\title{
Variação entre filhotes de representantes do complexo Bothrops neuwiedi.(Serpentes, Viperidae, Crotalinae)
}

\author{
Vinícius Xavier \\ Departamento de Zoologia, Instituto de Biociências, Universidade de Săo Paulo, Caixa Postal 11.461, CEP 05422-970, \\ São Paulo, SP.
}

\begin{abstract}
Variation among young individuals of Bothrops neuwiedi complex (Serpentes, Viperidae, Crotalinae). External morphological characters of 141 young specimens (69 males and 72 females) of the Bothrops neuwiedi complex were analyzed. Regression analysis was used in the study of morphometric characters and principal component analysis was used in the study of meristic and qualitative characters. Sexual dimorphism was confirmed in the meristic and morphometric characters. Males showed higher counts of subcaudals and longer tails. Females showed eventually higher number of ventrals and dorsal rows, and larger heads. Six different drawing patterns were diagnosed and can indicate the existence of different species. Ontogenetic variation was described.
\end{abstract}

Keywords: Bothrops neuwiedi complex, Crotalinae, Viperidae, Serpentes, Sexual dimorphism, geographic variation, ontogenetic variation.

Palavras-chave: Complexo Bothrops neuwiedi, Crotalinae, Viperidae, Serpentes, dimorfismo sexual, variação geográfica, variação ontogenética.

\section{Introdução}

O complexo Bothrops neuwiedi distribui-se amplamente pelas áreas abertas da América do Sul e é um dos grupos mais variáveis e taxonomicamente confusos dentro do gênero Bothrops, tendo sido alvo de uma recente revisão sistemática (Silva, 2000). Como nos demais grupos da subfamília Crotalinae, as ninhadas de representantes desse complexo taxonômico também foram abordadas principalmente sob o ponto de vista da história natural (Almeida, 1999; Alves et al., 1998; Leitão-de-Araújo et al., 1998; Melgarejo, 1977; Murphy e Mitchell, 1984; Peñaranda, 1995; Valdujo et al., no prelo) e da composição e ação do veneno (Castro, 1999; Furtado et al., 1991).

Recebido em 08.06.2001

Aceito em 02.09.2001
Para algumas espécies da subfamília já existem, portanto, dados preliminares nesse sentido, como Bothriechis aurifer, $B$. lateralis, $B$. marchi, B. nigroviridis, B. schlegelii (Antonio, 1980; Blody, 1983; Murphy e Mitchell, 1984), Atropoides nummifer (Cruz et al., 1989; Solórzano, 1989), Cerrophidion barbouri, C. godmani, Ophryacus melanurus (Murphy e Mitchell, 1984), Bothrops alternatus (Leitão-deAraújo e Matschulat-Ely, 1980; Leitão-de-Araújo e Perazzolo, 1974; Murphy e Mitchell, 1984; Pezzano, 1986), B. asper (Cruz et al., 1989; Solórzano e Cerdas, 1989), B. atrox (Hoge e Federsoni, 1978; Federsoni, 1980) e B. moojeni (Leloup, 1973).

Poucos trabalhos, contudo, foram dedicados a um enfoque sistemático da variação entre filhotes e/ou ninhadas dentro de Crotalinae. Uma análise preliminar foi feita com ninhadas de Crotalus durissus (Calleffo, 1996). Já foi de- 
monstrado para o grupo Bothrops atrox, por exemplo, a ocorrência de diferentes e complexos níveis de variações ontogenética, sexual e microgeográfica, que dificultam a análise estatística de caráter por caráter, considerada tradicional. Nesses casos, a análise multivariada temse revelado uma ferramenta extremamente útil (Wüster et al., 1996). Fazendo uso dessa ferramenta, o presente trabalho procurou interpretar a variação observada entre fillhotes (e algumas ninhadas) do complexo B. neuwiedi sob os enfoques sexual, ontogenético e geográfico.

\section{Material e Métodos}

Foram analisados 141 filhotes (69 machos e 72 fêmeas) do complexo B. neuwiedi (Apêndice I), distribuídos em 16 amostras de 14 localidades (Figura 1). Sete padrões de desenho foram diagnosticados dentro do complexo B. neuwiedi (PDs 1 a 7) (Silva, 2000), mas nenhum dos filhotes examinados apresentou o PD4. Os seis padrões observados no presente estudo estão sucintamente descritos a seguir:

- Padrão de desenho 1 (PD1 - Figura 2A, B) manchas com bordas difusas, sem margem branca ou com margem muito tênue, tornando as manchas pouco destacadas da coloração de fundo; manchas de coloração castanha, eventualmente enegrecidas, sobre fundo geralmente creme; manchas intercalares menores às vezes presentes entre as manchas dorsolaterais maiores; faixa pós-ocular pouco marcada; manchas dorsocefálicas posteriores não-alongadas, sem formar faixas dorsais atrás da cabeça; melanismo lateral da cabeça ausente; algumas escamas dorsais com carenas brancas destacadas da coloração de fundo; supralabiais com pequenas manchas brancas alongadas verticalmente, mais conspícuas nas supralabiais da metade posterior da boca.

- Padrão de desenho 2 (PD2 - Figura 2C) manchas com bordas bem definidas, com margem branca conspícua, tornando as manchas bastante destacadas da coloração de fundo; manchas de coloração castanho escura, sobre fundo castanho; manchas intercalares presentes entre as manchas dorsolaterais mai- ores; faixa pós-ocular marcada; manchas dorsocefálicas posteriores geralmente alongadas, formando faixas dorsais curtas atrás da cabeça; melanismo lateral da cabeça ausente; carenas com a mesma coloração de fundo das escamas dorsais; maioria das supralabiais de coloração castanho-escura com grandes manchas brancas geralmente arredondadas.

- Padrão de desenho 3 (PD3 - Figura 2D) manchas com bordas bem definidas ou não, com margem branca geralmente restrita à porção dorsolateral das manchas, que apresentam coloração em geral negra sobre fundo bege; manchas intercalares freqüentemente ausentes; faixa pós-ocular pouco marcada; manchas dorsocefálicas posteriores nãoalongadas, sem formar faixas dorsais atrás da cabeça; melanismo lateral da cabeça presente, faixa pós-ocular expandindo-se em torno da comissura labial, originando uma espécie de capuz melânico; carenas com a mesma coloração de fundo das escamas dorsais; maioria das supralabiais de coloração uniforme, sem manchas conspícuas.

- Padrão de desenho 5 (PD5 - Figura 2E) manchas com bordas bem definidas, com margem branca não muito conspícua; manchas de coloração castanho-escura ou negra, sobre fundo castanho-oliva; manchas intercalares presentes entre as manchas dorsolaterais maiores; faixa pós-ocular marcada; manchas dorso-cefálicas posteriores não-alongadas, sem formar faixas dorsais atrás da cabeça; melanismo lateral da cabeça ausente; carenas com a mesma coloração de fundo das escamas dorsais; supralabiais de coloração creme com grandes manchas castanho-escuras ou negras (cobrindo mais da metade da supralabial), mais conspícuas na metade anterior da boca, sendo que a maior mancha está localizada sempre sob o olho; eventualmente, tais manchas podem ocupar todas as supralabiais, originando o aspecto de pigmentação uniforme, como nas supralabiais do PD3.

- Padrão de desenho 6 (PD6 - Figura 2F) manchas com bordas bem definidas, com margem branca muito conspícua; manchas de coloração castanho-escura ou negra, sobre fundo geralmente castanho-terra; manchas intercalares presentes entre as manchas dor- 


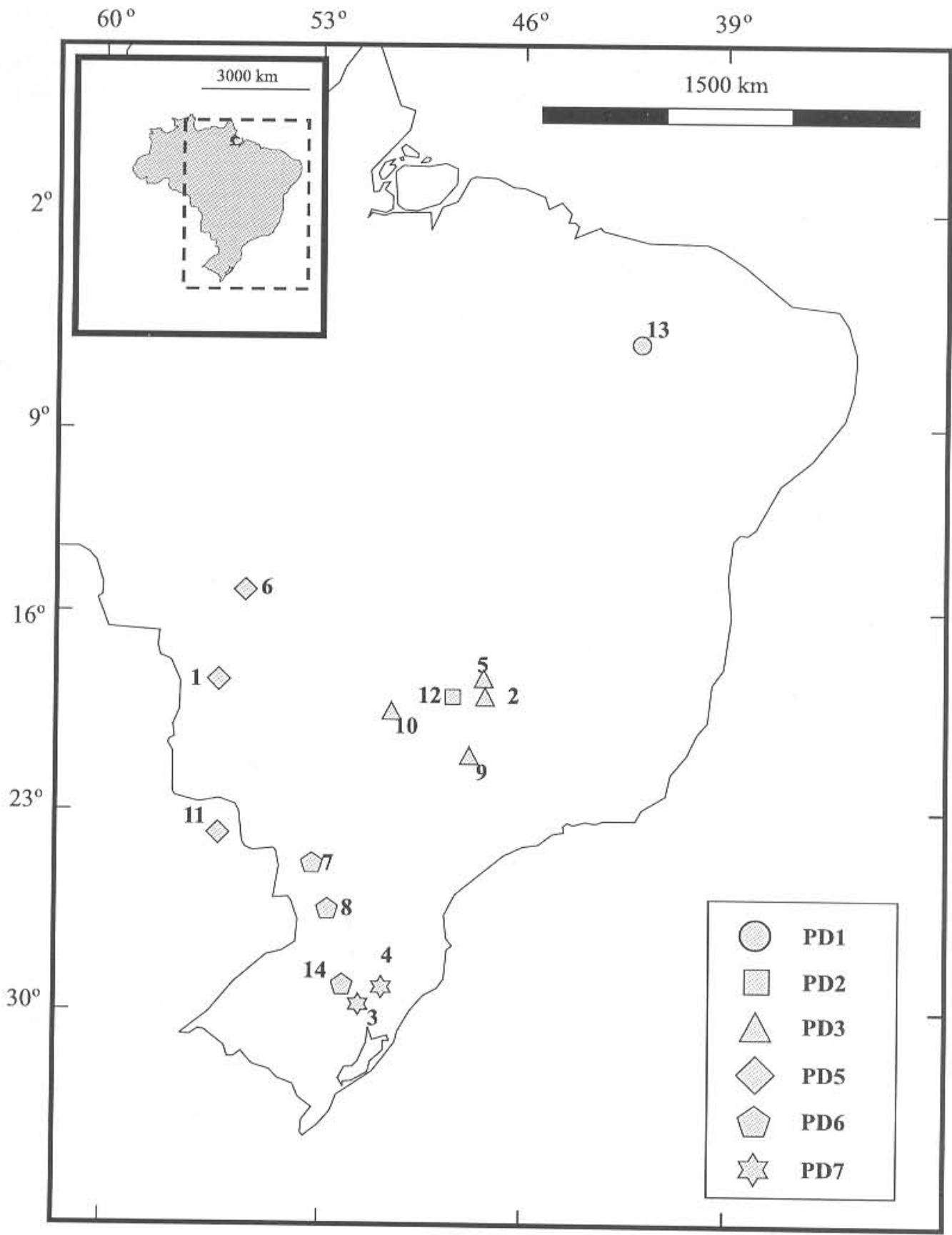

Figura 1 - Distribuição geográfica das amostras de filhotes do complexo Bothrops neuwiedi analisadas. Localidades: 1. Albuquerque, MS; 2. Araguari, MG; 3. Cachoeira do Sul, RS; 4. Capela de Santana, RS; 5. Catalāo, GO; 6. Cuiabá, MT; 7. Foz do Iguaçu, PR; 8. Itapiranga, SC; 9. Matão, SP; 10. Paranaíba, MS; 11. Pedro Juan Caballero, Paraguai; 12. Prata, MG; 13. Regeneração, PI; 14. Santa Cruz do Sul, RS. 
solaterais maiores; faixa pós-ocular marcada; manchas dorsocefálicas posteriores alongadas, formando faixas dorsais longas atrás da cabeça; melanismo lateral da cabeça ausente; carenas com a mesma coloração de fundo das escamas dorsais; supralabiais de coloração creme com grandes manchas castanho-escuras ou negras (cobrindo mais da metade da superfície da supralabial), mais conspícuas na metade anterior da boca, sendo que a maior mancha está localizada sempre sob o olho; eventualmente, tais manchas podem ocupar todas as supralabiais, originando o aspecto de pigmentação uniforme, como nas supralabiais do PD3.

- Padrão de desenho 7 (PD7 - Figura 2G) padrão com manchas de bordas bem definidas, com margem branca conspícua, tornando as manchas bastante destacadas da coloração de fundo; manchas de coloração castanho-escura ou negras, sobre fundo castanhoacinzentado; manchas intercalares presentes entre as manchas dorsolaterais maiores; faixa pós-ocular marcada; manchas dorsocefálicas posteriores geralmente alongadas, formando faixas dorsais curtas atrás da cabeça; melanismo lateral da cabeça ausente; carenas com a mesma coloração de fundo das escamas dorsais; maioria das supralabiais de coloração creme, com pequenas manchas castanhas triangulares (cobrindo menos da metade da superfície da supralabial), sempre na sutura entre duas supralabiais.

Foram analisados 16 caracteres da morfologia externa (quatro morfométricos, cinco merísticos e sete qualitativos), cujas descrições e abreviaturas são apresentadas a seguir.

\section{Caracteres morfométricos (em mm)}

1 - Comprimento da cabeça (CAB) - tomado com paquímetro (com precisão de $0,1 \mathrm{~mm})$, da escama rostral até a articulação quadrado-mandibular;

2 - Comprimento rostro-cloacal (CRC) tomado com escala milimetrada (com precisão de $1 \mathrm{~mm}$ ), da escama rostral até a placa cloacal;

3 - Comprimento da cauda (CAU) - tomado com escala milimetrada, da placa cloacal até a extremidade da cauda;
4 - Comprimento do tronco + cauda (TRCAU) - obtido da fórmula [(CRC - CAB $)+$ $\mathrm{CAU}]$; corresponde à distância da articulação quadrado-mandibular até a extremidade da cauda.

\section{Caracteres merísticos}

1 - $\mathrm{N}^{\circ}$ de escamas dorsais (D) - contadas nos terços anterior (D1) e mediano do tronco (D2);

2 - $\mathrm{N}^{\prime \prime}$ de escamas ventrais (V) - contadas a partir da primeira escama ventral mais larga que longa, excluindo-se a placa cloacal;

3 - No de escamas sub-caudais (SC) - contadas a partir das primeiras sub-caudais em contato até a extremidade da cauda, exceto o espinho terminal;

4 - $\mathrm{N}^{\prime \prime}$ de escamas que indicam a menor distância entre a mancha dorsolateral situada no meio do tronco e a mancha dorsolateral similar e anterior a ela (DA) (Figura 3 ).

Caracteres qualitativos:

1 - Coloração da carena (CAR) - carena da mesma cor da escama (estado 0); carena branca (estado 1);

2 - Manchas intercalares (MI) - presentes (0) (Figura 3); ausentes (1);

3 - Bordas das manchas dorsais (BMD) com contornos bem definidos, tornando as manchas destacadas da coloração de fundo $(0)$ (Figura 3); difusas, destacando pouco as manchas da coloração de fundo (1) (Figura 2B);

4 - Faixa(s) pós-cefálica(s) (FPC) - ausente(s) $(0)$; presente(s) e de comprimento menor ou igual ao comprimento das manchas occipitais (1); presente(s) e de comprimento maior que o das manchas occipitais (2) (Figura 2F);

5 - Melanismo na região látero-ventral da cabeça (MEL) - ausente (0); presente (1) (Figura $2 \mathrm{D})$.

6 - Pigmentação das supralabiais (PSL) manchas ausentes, pigmentação uniforme $(0)$ (Figura 2D); grandes manchas brancas, geralmente arredondadas, sobre fundo castanho-escuro a negro (1) (Figura 2C); pequenas manchas brancas alongadas verticalmente sobre fundo castanho (2) (Figura 2A); grandes manchas castanho-escuras (cobrindo mais de $50 \%$ da super- 

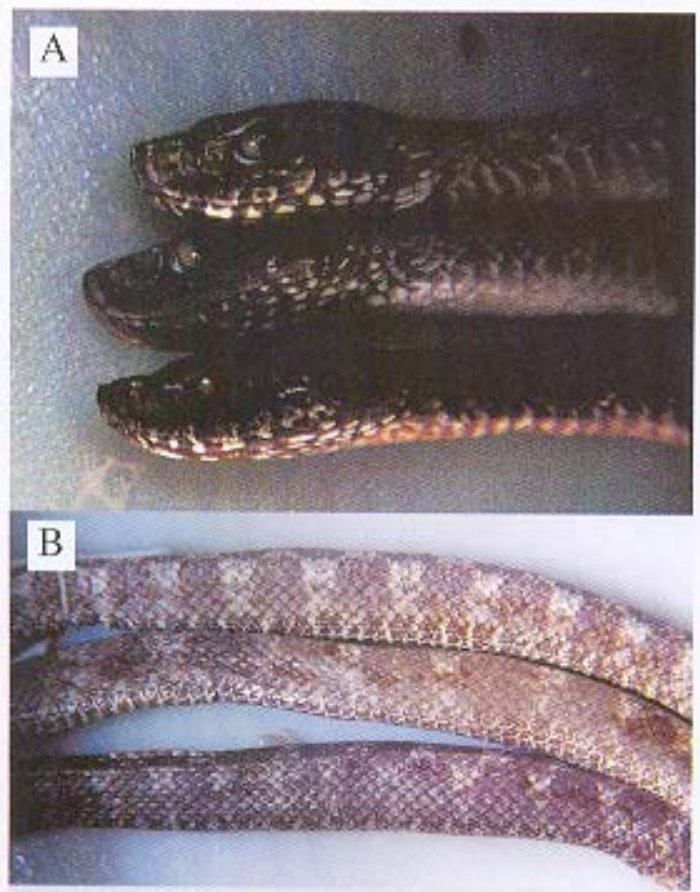

C

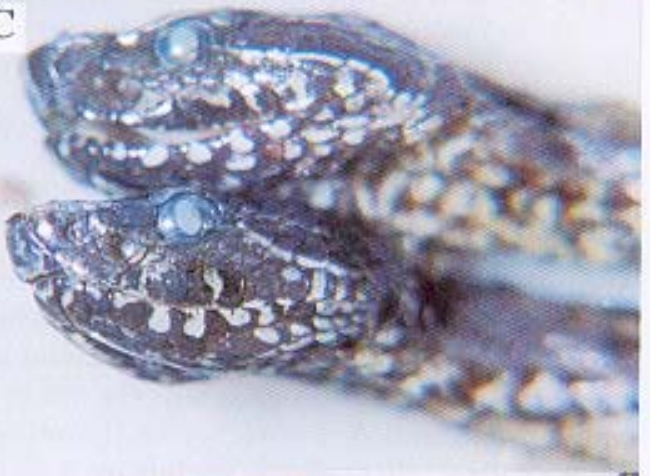

D

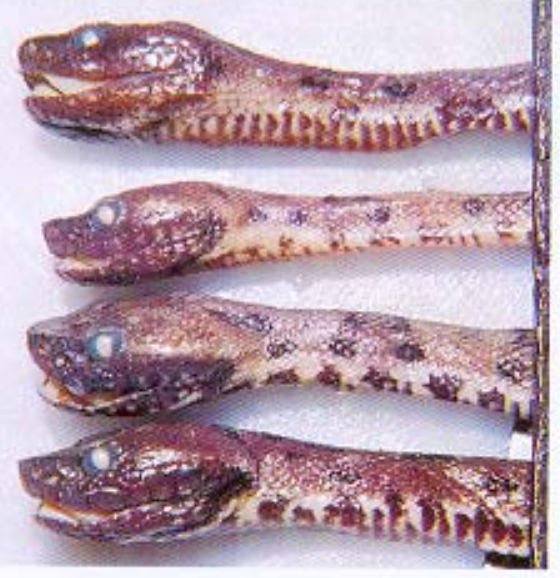

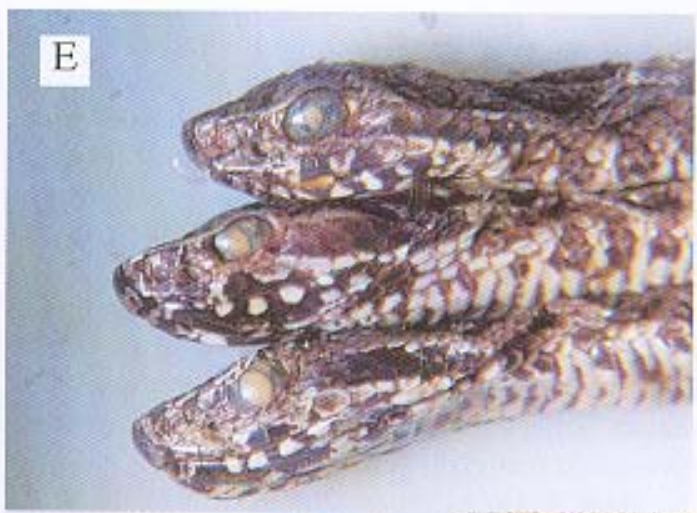

$\mathrm{F}$
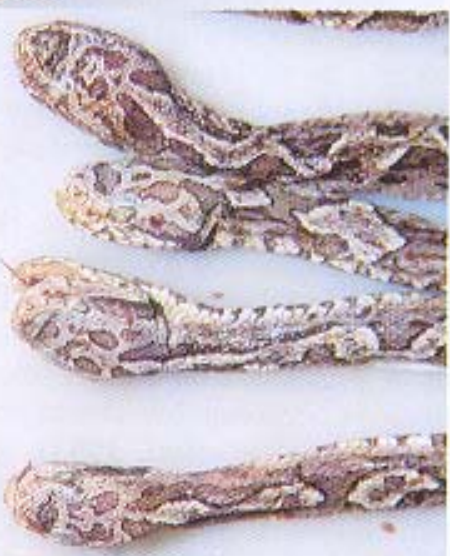

G

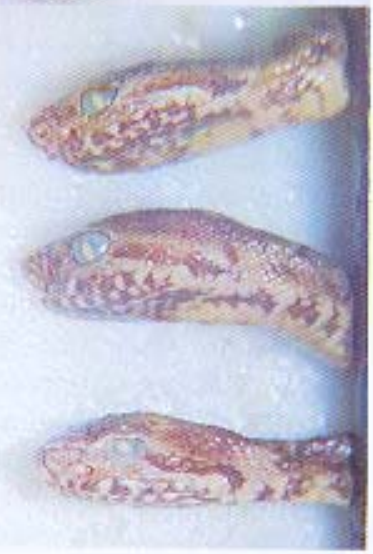

Figura 2 - Filhotes de algumas das amostras do complexo Bothrops nenwiedi analisadas. A e B) Regeneraçăo, PI (PD1); C) Prata, MG (PD2); D) Matāo, SP (PD3b); E) Albuquerque, MS (PD5b); F) Santa Cruz do Sul, RS (PD6d); G) Capela de Santana, RS (PD7a). A, C, D, E e G $=$ vista lateral da cabeça; $\mathbf{B}=$ vista lateral do tronco e $\mathrm{F}=$ vista dorsal da cabeça. 
fície da supralabial) sobre fundo creme, a maior mancha localizada exatamente sob o olho (3) (Figura 2E); manchas castanhas triangulares sobre fundo creme (geralmente cobrindo menos de $50 \%$ da superfície da supralabial), sempre nas suturas entre duas supralabiais (4) (Figura 2G);

7 - Extensão da pigmentação das supralabiais (EPSL) - presente em todas as supralabiais (0) (Figura 2C, D, G); mais conspícua na metade anterior (1) (Figura 2E); mais conspícua na metade posterior (2) (Figura 2A).

Para testar estatisticamente a ocorrência de dimorfismo sexual em todos os caracteres merísticos, empregou-se o teste $t$, com um nível de significância de 0,05 . Esse teste é bastante robusto, mesmo para distribuições não-normais (Vanzolini, 1993) e foi feito no programa SYSTAT versão 5.03 (Systat, 1993).

Foi registrada a estatística descritiva de três caracteres morfométricos (CAB, CRC e CAU), considerando-se machos e fêmeas separadamente. A existência de dimorfismo sexual nas proporções corporais foi testada por uma análise de regressão (Vanzolini, 1993), teste considerado robusto mesmo quando alguns de seus requisitos não são obedecidos (Zar, 1984). Foram analisadas basicamente duas regressões: 1) o comprimento da cabeça (CAB) foi definido como variável dependente (y), tendo o comprimento do tronco + cauda (TRCAU) como variável independente $(x)$ e 2) o comprimento da cauda (CAU) teve o comprimento rostro-cloacal (CRC) como variável independente. A escolha dessas variáveis teve como objetivo evitar a auto-correlação (Vanzolini, 1993). Os gráficos obtidos no programa SYSTAT versão 5.03 (Systat, 1993) geralmente indicaram regressões lineares, e algumas anamorfoses (arit-log, logarit e log-log, para os valores de x e y) não modificaram significativamente os coeficientes de determinação, sendo então ignoradas. Para verificar se as diferenças entre essas regressões eram estatisticamente significativas, foi utilizado o teste $t(\mathrm{p}=0,05)$ para coeficientes (b) e constantes de regressão (a), segundo Vanzolini (1993) e Zar (1984).

Os caracteres qualitativos (MI, BMD, FPC, MEL, OSL e EOSL) e merísticos (D2 e DA) mais informativos, segundo análises prévias, ti-

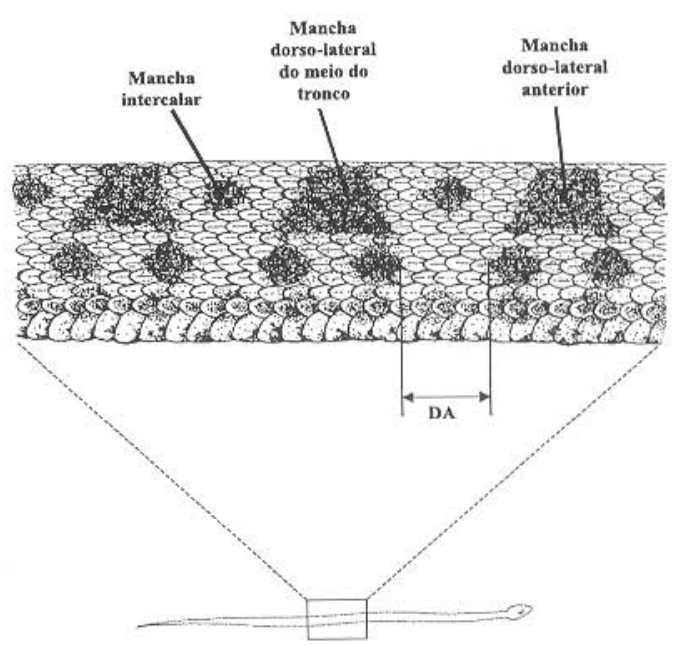

Figura 3 - Vista lateral do meio do tronco de um filhote do complexo Bothrops neuwiedi. Podem ser observadas: as bordas das manchas dorso-laterais de contorno definido (estado 0 do caráter BMD), a presença de manchas intercalares entre as manchas dorso-laterais maiores (estado 0 do caráter MI) e a distância de quatro escamas entre a mancha dorsolateral do meio do tronco e sua similar anterior (caráter DA).

veram sua variação interpretada por meio da análise de componentes principais (Crisci e Armengol, 1983; James e McCulloch, 1990; Thorpe, 1976, 1980). Os sexos foram estudados separadamente, e cada indivíduo constituiu uma unidade taxonômica operacional. Os dados foram padronizados e, a partir deles, foram obtidas as matrizes de distância euclideana e de correlação de Pearson, que possibilitaram a análise multivariada. Todo esse procedimento foi feito com o programa SYSTAT versão 5.03 (Systat, 1993).

\section{Resultados}

Dezesseis amostras de filhotes foram examinadas (Tabela 1). Duas localidades (Foz do Iguaçu - PR e Cachoeira do Sul - RS) contribuíram com duas amostras cada uma. Optou-se por tratar essas amostras separadamente devido aos números de coleção discrepantes (amostras tombadas na coleção em épocas diferentes) (Apên- 
dice I). Dessa forma, foi possível comparar, dentro da mesma localidade, amostras diferentes com o mesmo padrão de desenho. Os caracteres merísticos que apresentaram dimorfismo sexual dentro de certas amostras são apresentados na Tabela 1.

As freqüencias relativas dos caracteres merísticos que indicaram dimorfismo sexual em algumas das amostras foram representadas em histogramas (Figuras 4 e 5). De modo geral, houve uma tendência de dimorfismo no número de sub-caudais, ventrais e dorsais. Os machos apresentaram comparativamente mais sub-caudais que as fêmeas, as quais, por sua vez, mostraram maiores contagens de ventrais e de dorsais (Figuras 4 e 5 ). Além do dimorfismo se- xual, os histogramas das Figuras 4 e 5 também registram uma parcela da ampla variação geográfica presente no complexo B. neuwiedi.

A estatística descritiva dos três caracteres morfométricos (CAB, CRC e CAU) pode ser observada nos Apêndices II a IV, respectivamente. Os machos de todas as amostras apresentaram comprimento médio da cauda maior que o das respectivas fêmeas (Apêndice IV). Estas, por sua vez, apresentaram comprimento médio da cabeça maior que o dos machos na maioria das amostras (Apêndice II), mas não repetiram a mesma tendência na média do comprimento rostro-cloacal (Apêndice III). Isto coincide com os dados merísticos (Tabela 1), onde o número de sub-caudais (uma indi-

Tabela 1 - Número de filhotes do complexo Bothrops neuwiedi analisados em 16 amostras e os respectivos caracteres merísticos que indicaram dimorfismo sexual significativo ao nível de $5 \%$, segundo o teste $t$. PD $=$ padrão de desenho, $t=$ coeficiente resultante do teste $t$, g. 1. = graus de liberdade. As amostras marcadas com asterisco $(*)$ constituem ninhadas efetivas, das quais se conhece a mãe. Para a abreviatura dos caracteres, ver item Material e Métodos.

\begin{tabular}{|c|c|c|c|c|c|c|c|c|}
\hline \multirow{2}{*}{ Amostras } & \multirow{2}{*}{ PD } & \multicolumn{3}{|c|}{$\mathbf{N}$} & \multirow{2}{*}{$\begin{array}{l}\text { Caracteres } \\
\text { Merísticos }\end{array}$} & \multirow[t]{2}{*}{$t$} & \multirow[t]{2}{*}{ g. 1.} & \multirow[t]{2}{*}{$\mathbf{P}$} \\
\hline & & M & $\mathbf{F}$ & $\mathbf{T}$ & & & & \\
\hline Regeneração, PI & 1 & 3 & 2 & 5 & - & - & - & - \\
\hline Prata, $\mathrm{MG}$ & 2 & 2 & 3 & 5 & - & - & - & - \\
\hline \multirow[t]{2}{*}{ Paranaíba, MS } & $3 a$ & 4 & 4 & 8 & $\mathrm{SC}$ & 4,176 & 6 & $0,001<\mathrm{P}<0,01$ \\
\hline & & & & & DA & 2,611 & 6 & $0,01<\mathrm{P}<0,05$ \\
\hline Matão, SP* & $3 b$ & 3 & 5 & 8 & $\mathrm{SC}$ & 3,798 & 6 & $0,001<\mathrm{P}<0,01$ \\
\hline Catalão, GO & $3 c$ & 3 & 4 & 7 & - & - & - & - \\
\hline Araguari, $\mathrm{MG}^{*}$ & $3 d$ & 2 & 4 & 6 & - & - & - & - \\
\hline Cuiabá, MT & $5 \mathrm{a}$ & 6 & 3 & 9 & V & 2,640 & 7 & $0,01<\mathrm{P}<0,05$ \\
\hline \multirow[t]{3}{*}{ Albuquerque, MS* } & $5 \mathrm{~b}$ & 9 & 14 & 23 & $\mathrm{~V}$ & 2,979 & 21 & $0,001<\mathrm{P}<0,01$ \\
\hline & & & & & $\mathrm{SC}$ & 7,478 & 21 & $\mathrm{P}<0,001$ \\
\hline & & & & & D1 & 7,201 & 6 & $\mathrm{P}<0,001$ \\
\hline \multirow[t]{2}{*}{ Pedro Juan Caballero, PAR* } & $5 c$ & 4 & 4 & 8 & D2 & 2,828 & 6 & $0,01<\mathrm{P}<0,05$ \\
\hline & & & & & $\mathrm{SC}$ & 5,417 & 6 & $0,001<\mathrm{P}<0,01$ \\
\hline \multirow[t]{2}{*}{ Foz do Iguaçu, PR (1) } & $6 \mathrm{a}$ & 5 & 2 & 7 & - & - & - & - \\
\hline & & & & & D1 & 3,466 & 16 & $0,001<\mathrm{P}<0,01$ \\
\hline \multirow{2}{*}{ Foz do Iguaçu, PR (2) } & $6 \mathrm{~b}$ & 12 & 6 & 18 & D2 & 6,144 & 16 & $\mathrm{P}<0,001$ \\
\hline & & & & & $\mathrm{SC}$ & 2,886 & 16 & $0,01<\mathrm{P}<0,05$ \\
\hline Itapiranga, $\mathrm{SC}^{*}$ & $6 c$ & 3 & 4 & 7 & $\mathrm{SC}$ & 2,688 & 5 & $0,01<\mathrm{P}<0,05$ \\
\hline \multirow[t]{2}{*}{ Santa Cruz do Sul, RS* } & $6 \mathrm{~d}$ & 7 & 8 & 15 & D1 & 3,984 & 13 & $0,001<\mathrm{P}<0,01$ \\
\hline & & & & & $\mathrm{SC}$ & 6,737 & 13 & $0,001<\mathrm{P}<0,01$ \\
\hline \multirow[t]{2}{*}{ Capela de Santana, RS* } & $7 a$ & 5 & 5 & 10 & D1 & 4,000 & 8 & $0,001<\mathrm{P}<0,01$ \\
\hline & & & & & D2 & 2,530 & 8 & $0,01<\mathrm{P}<0,05$ \\
\hline Cachoeira do Sul, RS (1) & $7 \mathrm{~b}$ & 1 & 2 & 3 & - & - & - & - \\
\hline Cachoeira do Sul, RS (2) & $7 \mathrm{c}$ & - & 2 & 2 & - & - & - & - \\
\hline TOTAL & - & 69 & 72 & 141 & - & - & - & - \\
\hline
\end{tabular}


cação indireta do comprimento relativo da cauda) foi mais preponderante na indicação do dimorfismo sexual que o número de ventrais (uma indicação indireta do comprimento relativo do corpo).

Um estudo mais preciso, porém, pode ser obtido a partir das análises de regressão (Tabela 2). Os parâmetros de tais análises indicaram que apenas a amostra de Albuquerque - MS apresentou as duas regressões analisadas estatisticamente significativas em ambos os sexos.

$\mathrm{Na}$ amostra de Albuquerque, foram testadas as diferenças entre machos e fêmeas pelo teste $t$ para coeficientes e constantes das duas regressões analisadas $(p=0,05)$. Tanto na regressão CAB $\times$ TRCAU $\left(\mathrm{t}_{\mathrm{a}}=2.733 ; \mathrm{g} .1 .=19 ; 0,01<\mathrm{P}\right.$ $<0,05)$ como na regressão CAU x CRC $\left(\mathrm{t}_{\mathrm{a}}=\right.$ $4,104 ;$ g. 1. $=19 ; \mathrm{p}<0,001)$, o teste $t$ indicou que as constantes de regressão mostraram-se estatisticamente diferentes, corroborando o dimorfismo sexual: fêmeas com cabeças maiores e caudas mais curtas que os machos (Figura 6).

Com relação à análise de componentes principais, as Tabelas 3 e 4 trazem os auto-valores para os três primeiros componentes principais, a porcentagem da variação total representada por cada auto-valor e as porcentagens acumuladas respectivamente para machos e fêmeas das amostras analisadas.

Em ambos os sexos, os três primeiros componentes principais expressaram mais de $80 \%$ da variação entre os caracteres merísticos e qualitativos analisados (Tabelas 3 e 4). Duas combinações desses três primeiros componentes principais $\left(1^{ \pm} \times 2^{\underline{a}}\right.$ e $\left.1^{\circ} \times 3^{\circ}\right)$ foram mais informativas na representação das relações de similaridade entre os filhotes de cada sexo. Tais combinações foram projetadas separadamente para machos e fêmeas em gráficos bidimensionais (Figura 7). As seis cores representadas correspondem aos seis padrões de desenho (PDs) presentes nas amostras analisadas. Símbolos diferentes da mesma cor correspondem às diferentes amostras com o mesmo PD. A combinação dos dois primeiros componentes principais discriminou melhor as amostras entre si, tanto nos machos, como nas fêmeas.

De um modo geral, em ambos os sexos, amostras diferentes com o mesmo PD formaram grupos homogêneos e mais próximos entre si do que em relação às amostras com outros PDs. Na combinação dos componentes principais 1 e 3, a separação entre os diferentes PDs foi menos nítida, principalmente entre as amostras com os PDs 5 (amarelo), 6 (verde) e 7 (preto). As amostras com o PD5 foram as que apresentaram maior dispersão dos indivíduos (Figura 7), o que pode ser uma evidência da maior heterogeneidade ou variação dessas amostras.

As análises de componentes principais em machos e fêmeas (Tabelas 5 e 6) mostraram pequenas diferenças entre si, que corroboraram a necessidade de se tratar os sexos separadamente.

Ao longo do eixo 1 (111 componente principal), em ambos os sexos, as amostras com o PD3 distinguiram-se de todas as demais (Figura 7). Entre os caracteres que mais contribuíram para esse eixo, tanto para machos como para fêmeas, estão MI, BMD, MEL e PSL (Tabelas 5 e 6 ). Estes caracteres permitiram diagnosticar o PD3: manchas intercalares ausentes (estado 1 do caráter MI), borda das manchas dorsais difusas (estado 1 do caráter BMD), presença de melanismo látero-ventral na cabeça (estado 1 do caráter MEL; Figura 2D) e supralabiais uniformemente pigmentadas, sem manchas conspícuas (estado 0 do caráter PSL; Figura 2D).

Nesse primeiro eixo, responsável pela representação da maior parte da variação observada (Tabelas 3 e 4), também foi possível distinguir os PDs 1 e 2 dos demais. O caráter que provavelmente mais influenciou nesse resultado foi o tipo de pigmentação das supralabiais (PSL), que, no PD1, apresenta o estado 2 (Figura 2A) e, no PD2, o estado 1 (Figura 2C). A amostra com o PD1 apresentou ainda dois caracteres com os mesmos estados observados nas amostras com o PD3: manchas intercalares ausentes ou pouco conspícuas (estado 1 do caráter MI; Figura 2B) e bordas das manchas dorsais difusas (estado 1 do caráter BMD; Figura 2B). Ao longo desse eixo, amostras com os PDs 6 e 7 também mostraram diferenças entre si e com relação aos PDs 1, 2 e 3. As amostras com o PD5, por sua vez, aparecem sobrepostas aos PDs $6 \mathrm{e}$ 7 nesse eixo, mas distinguem-se dos PDs 1, 2 e 3. Novamente, o caráter PSL apresentou estados exclusivos nesses padrões: as amostras com o PD6 (assim como aquelas com o PD5) possuem 
Tabela 2 - Parâmetros das regressōes CAB x TRCAU e CAU x CRC para machos e fêmeas das amostras de filhotes analisadas dentro do complexo Bothrops neuwiedi que apresentaram alguma regressão significativa ao nível de $10 \%$. $\mathrm{a}=$ constante da regressão, $\mathrm{b}=$ coeficiente da regressão, $\mathrm{F}=$ quociente de variâncias de Fisher, $\mathrm{p}=$ significância da regressão.

\begin{tabular}{|c|c|c|c|c|c|}
\hline Regr./Sexo & Ninhadas & $\mathbf{a}$ & b & $\mathbf{F}$ & $\mathbf{P}$ \\
\hline \multirow{3}{*}{$\begin{array}{c}\text { CAB } x \text { TRCAU } \\
\sigma^{\pi}\end{array}$} & Albuquerque, MS & 4,899 & 0,042 & 8,040 & 0,025 \\
\hline & Foz do Iguaçu, PR (1) & 10,512 & 0,022 & 7,559 & 0,071 \\
\hline & Santa Cruz do Sul, RS & 6,863 & 0,077 & 17,902 & 0,008 \\
\hline \multirow{3}{*}{$\begin{array}{c}\text { CAU x CRC } \\
\sigma^{x}\end{array}$} & Albuquerque, MS & 13,805 & 0,109 & 6,115 & 0,043 \\
\hline & Pedro Juan Caballero, PAR & 91,479 & 0,561 & 57,933 & 0,017 \\
\hline & Foz do Iguaçu, PR (1) & 13,950 & 0,218 & 42,717 & 0,007 \\
\hline \multirow{3}{*}{$\begin{array}{c}\text { CAB x TRCAU } \\
q \\
7\end{array}$} & Matão, SP & 3,855 & 0,048 & 43,400 & 0,007 \\
\hline & Albuquerque, MS & 2,817 & 0,055 & 30,123 & 0,000 \\
\hline & Capela de Santana, RS & 19,028 & 0,162 & 40,370 & 0,008 \\
\hline \multirow{4}{*}{$\begin{array}{c}\mathrm{CAU} \times \mathrm{CRC} \\
\text { 우 }\end{array}$} & Prata, MG & 49,327 & 0,086 & 176,333 & 0,048 \\
\hline & Matão, SP & 18,084 & 0,218 & 108,481 & 0,002 \\
\hline & Albuquerque, MS & 15,957 & 0,081 & 3,810 & 0,077 \\
\hline & Foz do Iguaçu, PR (2) & 4,847 & 0,112 & 7,520 & 0,052 \\
\hline
\end{tabular}

Tabela 3 - Auto-valores dos três primeiros componentes principais e as respectivas porcentagens da variação total e porcentagens acumuladas para os machos das amostras de filhotes do complexo Bothrops neuwiedi.

\begin{tabular}{cccc}
\hline Componente principal & Auto-valor & $\begin{array}{c}\text { Porcentagem da } \\
\text { variação total }\end{array}$ & $\begin{array}{c}\text { Porcentagem } \\
\text { acumulada }\end{array}$ \\
\hline $\mathbf{1}^{\mathbf{0}}$ & 33,3 & 48,2 & 48,2 \\
$\mathbf{2}^{\mathbf{9}}$ & 15,3 & 22,2 & 70,4 \\
$\mathbf{3}^{\mathbf{0}}$ & 8,5 & 12,3 & 82,7 \\
\hline
\end{tabular}

Tabela 4 - Auto-valores dos três primeiros componentes principais e as respectivas porcentagens da variação total e porcentagens acumuladas para as fêmeas das amostras de filhotes do complexo Bothrops neuwiedi.

\begin{tabular}{cccc} 
Componente principal & Auto-valor & $\begin{array}{c}\text { Porcentagem da } \\
\text { variação total }\end{array}$ & $\begin{array}{c}\text { Porcentagem } \\
\text { acumulada }\end{array}$ \\
\hline $\mathbf{1}^{\mathbf{0}}$ & 37,9 & 52,6 & 52,6 \\
$\mathbf{2}^{\mathbf{a}}$ & 12,5 & 17,4 & 70,0 \\
$\mathbf{3}^{\text {o }}$ & 9,8 & 13,7 & 83,7 \\
\hline
\end{tabular}

Tabela 5 - Contribuições (independentemente do sinal) dos caracteres merísticos e qualitativos analisados para cada um dos três primeiros componentes principais para os machos das amostras de filhotes do complexo Bothrops neuwiedi. Os valores em negrito correspondem às três maiores contribuiçôes dentro de cada componente principal. Para a abreviatura dos caracteres, ver item Material e Métodos.

\begin{tabular}{cccc}
\hline Caráter & $\mathbf{1}^{\mathbf{0}}$ componente principal & $\mathbf{2}^{\text {- }}$ componente principal & 3⿻- componente principal $^{\text {- }}$ \\
\hline MI & $\mathbf{0 , 9 3 8}$ & 0,165 & 0,246 \\
BMD & $\mathbf{0 , 9 3 8}$ & 0,165 & 0,246 \\
MEL & $\mathbf{0 , 9 3 4}$ & $-0,197$ & 0,201 \\
PSL & $-0,920$ & 0,067 & $\mathbf{- 0 , 2 5 5}$ \\
DA & 0,708 & $-0,215$ & $\mathbf{- 0 , 4 7 9}$ \\
FPC & $-0,609$ & $\mathbf{- 0 , 2 8 2}$ & $\mathbf{0 , 4 8 2}$ \\
EPSL & $-0,587$ & $\mathbf{0 , 6 5 7}$ & 0,241 \\
D2 & $-0,470$ & $\mathbf{- 0 , 6 4 2}$ & 0,233
\end{tabular}


Freq. (\%) D1 - Pedro Juan Caballero, PAR (PD5a)

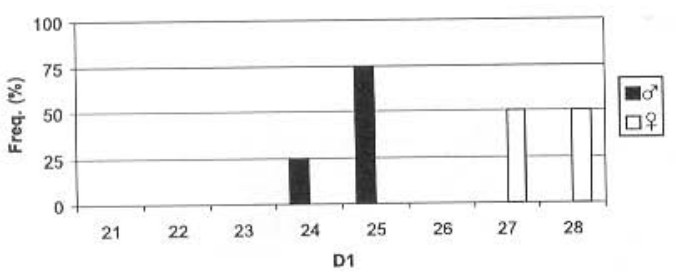

Freq. (\%) D1 - Foz do Iguaçu, PR(2) (PD6b)

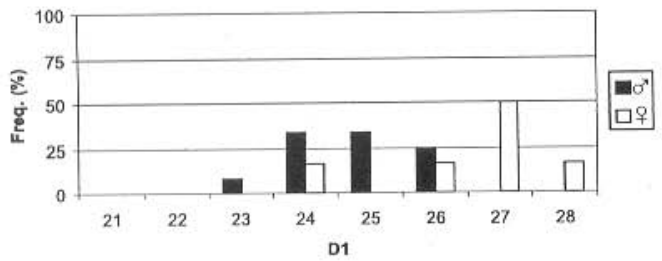

Freq. (\%) D1 - Santa Cruz do Sul, RS (PD6d)

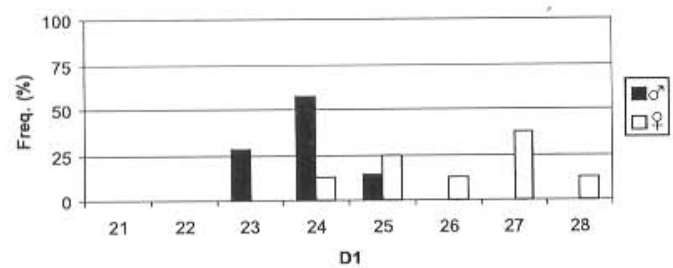

Freq. (\%) D1 - Capela de Santana, RS (PD7a)

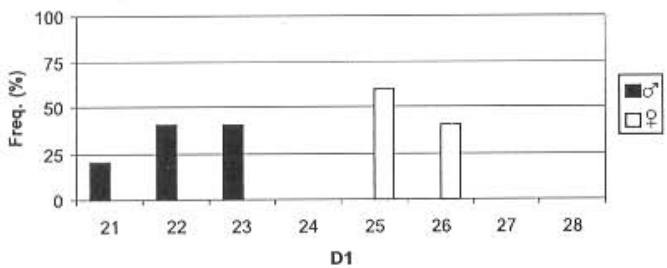

Freq. (\%) D2 - Pedro Juan Caballero, PAR (PD5c)

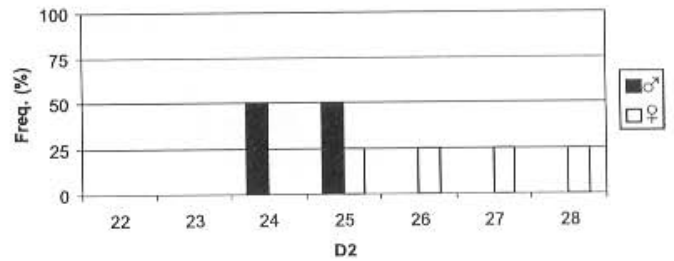

Freq. (\%) D2 - Foz do Iguaçu, PR (2) (PD6b)

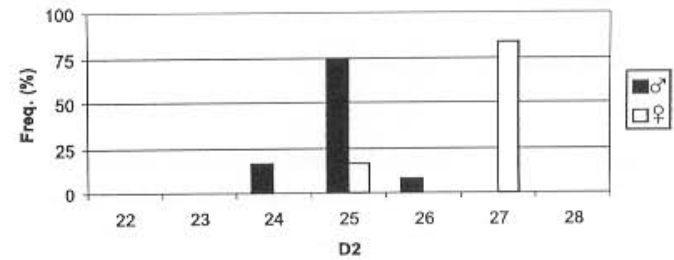

Freq. (\%) D2 - Capela de Santana, RS (PD7a)

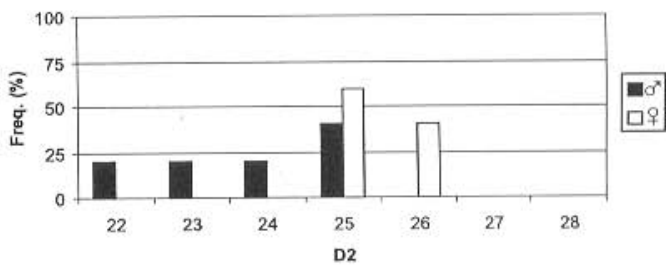

Figura 4 - Freqüências relativas do número de dorsais nos terços inicial (D1) e mediano (D2) do tronco para machos e fêmeas de algumas amostras de filhotes do complexo Bothrops neuwiedi analisadas. 
Freq. (\%) V - Cuiabá, MT (PD5a)

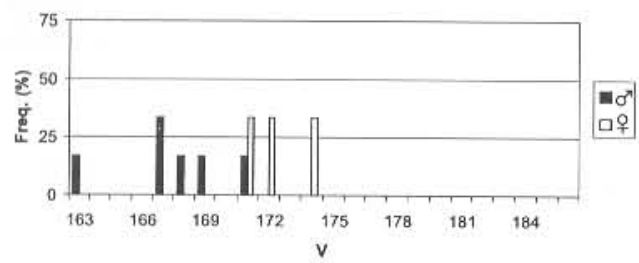

Freq. (\%) V - Albuquerque, MS (PD5b)

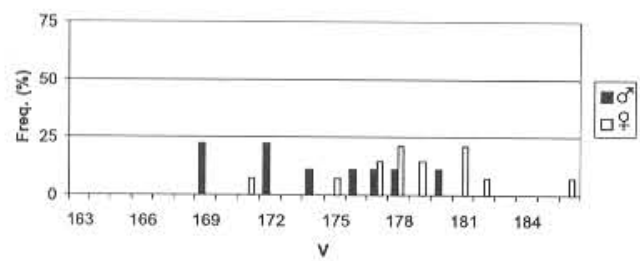

Freq. (\%) SC - Paranaíba, MS (PD3a)
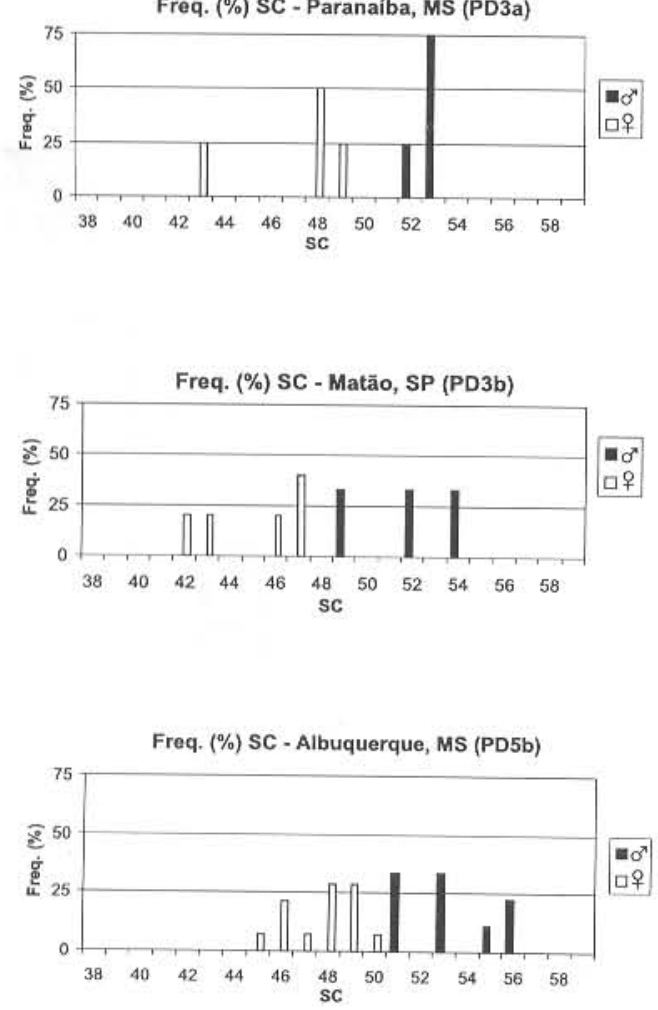

Freq. (\%) SC - Pedro Juan Caballero, PAR (PD5c)

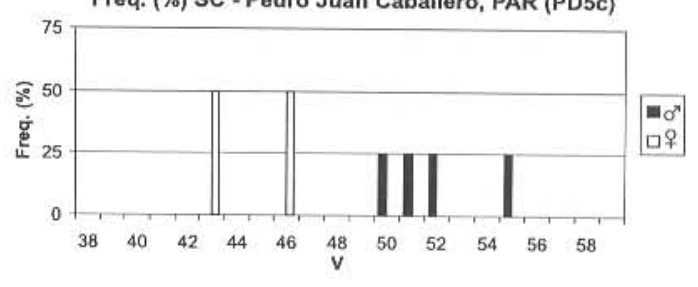

Freq. (\%) SC - Foz do Iguaçu, PR (2) (PD6b)
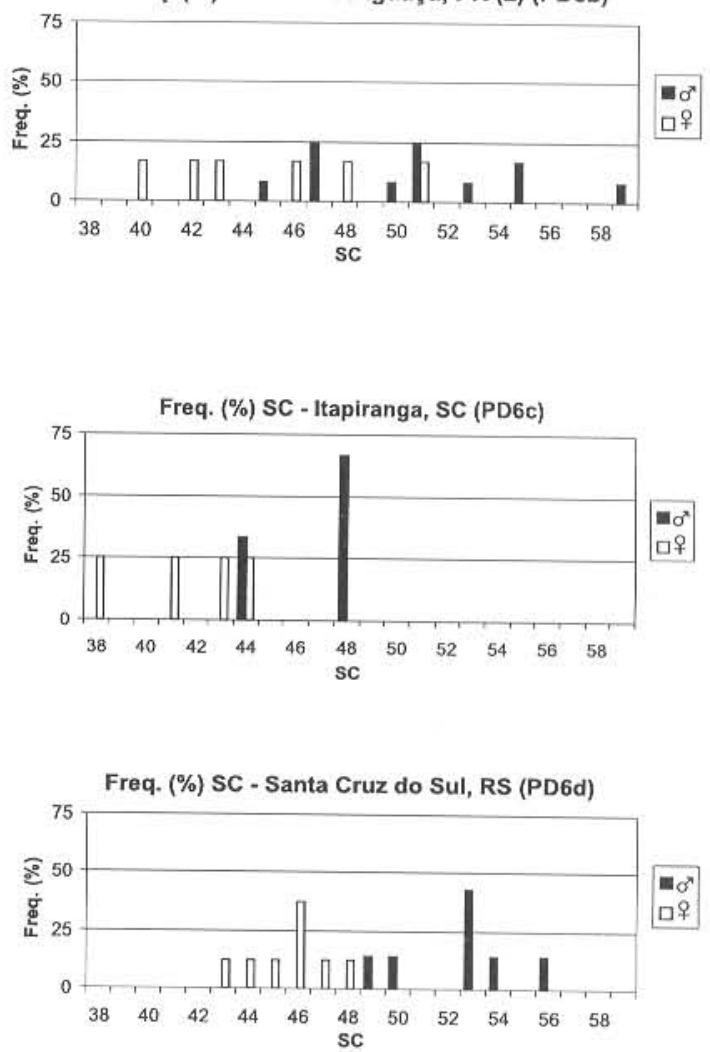

Figura 5 - Freqüências relativas do número de ventrais (V) e de sub-caudais (SC) para machos e fêmeas de algumas amostras de filhotes do complexo Bothrops neuwiedi analisadas. 
Tabela 6 - Contribuições (independentemente do sinal) dos caracteres merísticos e qualitativos analisados para cada um dos três primeiros componentes principais para as fêmeas das amostras de filhotes do complexo Bothrops neuwiedi, Os valores em negrito correspondem às três maiores contribuições dentro de cada componente principal. Para a abreviatura dos caracteres, ver item Material e Métodos.

\begin{tabular}{cccc}
\hline Caráter & $\mathbf{1}^{\mathbf{0}}$ componente principal & $\mathbf{2}^{\underline{0}}$ componente principal & $\mathbf{3}^{\mathbf{0}}$ componente principal \\
\hline MEL & $\mathbf{0 , 9 4 7}$ & 0,057 & 0,248 \\
MI & $\mathbf{0 , 9 4 0}$ & $-0,090$ & 0,270 \\
BMD & $\mathbf{0 , 9 4 0}$ & $-0,090$ & 0,270 \\
PSL & $-0,931$ & 0,071 & $-0,182$ \\
EPSL & $-0,651$ & $\mathbf{- 0 , 5 6 5}$ & 0,205 \\
FPC & $-0,600$ & 0,310 & $\mathbf{0 , 5 0 8}$ \\
DA & 0,591 & $\mathbf{0 , 5 0 2}$ & $\mathbf{- 0 , 4 7 4}$ \\
D2 & $-0,486$ & $\mathbf{0 , 6 1 3}$ & $\mathbf{0 , 3 9 8}$ \\
\hline
\end{tabular}

o estado 3 desse caráter (Figura 2E) e as amostras com o PD7, o estado 4 (Figura 2G).

Ao longo do segundo componente principal, é possível visualizar a segregação das amostras com os PDs 1 e 5 das demais amostras. Entre os caracteres que mais contribuíram em ambos os sexos para esse componente estão: o número de dorsais no meio do tronco (D2), o número de escamas que indicam a menor distância entre a mancha dorsolateral situada no meio do tronco e a mancha dorsolateral similar e anterior a ela (DA), o tipo de faixas pós-cefálicas (FPC) e a

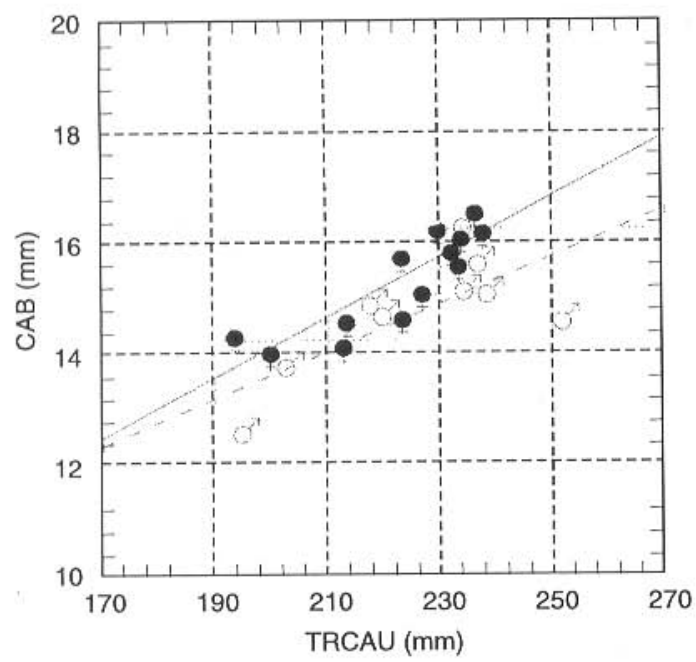

extensão da pigmentação das supralabiais (EPSL) (Tabelas 5 e 6). O caráter D2 nos PDs 1 e 5 não difere significativamente dos demais, e o caráter DA sequer pôde ser registrado para as amostras com o PD1 devido à fraca delimitação das manchas dorsolaterais (Figura 2B). O caráter EPSL exerceu importante papel na segregação das amostras com o PD1. São as únicas amostras nas quais a pigmentação das supralabiais é mais conspícua na metade posterior da boca (estado 2 do caráter EPSL; Figura 2A). O mesmo caráter nas amostras com os PDs 5 e 6

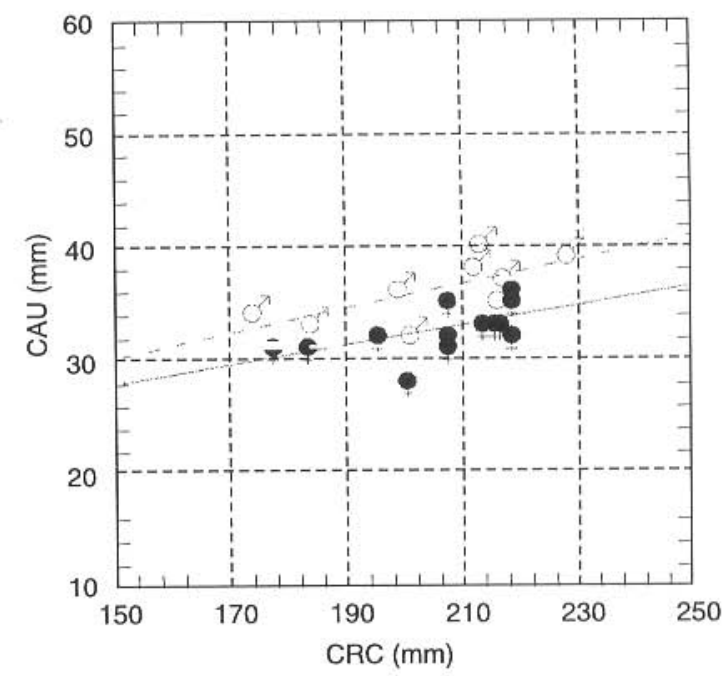

Figura 6 - Regressões CAB x TRCAU e CAU x CRC para machos e fêmeas da amostra de filhotes do complexo Bothrops neuwiedi de Albuquerque, MS (PD5b) . 

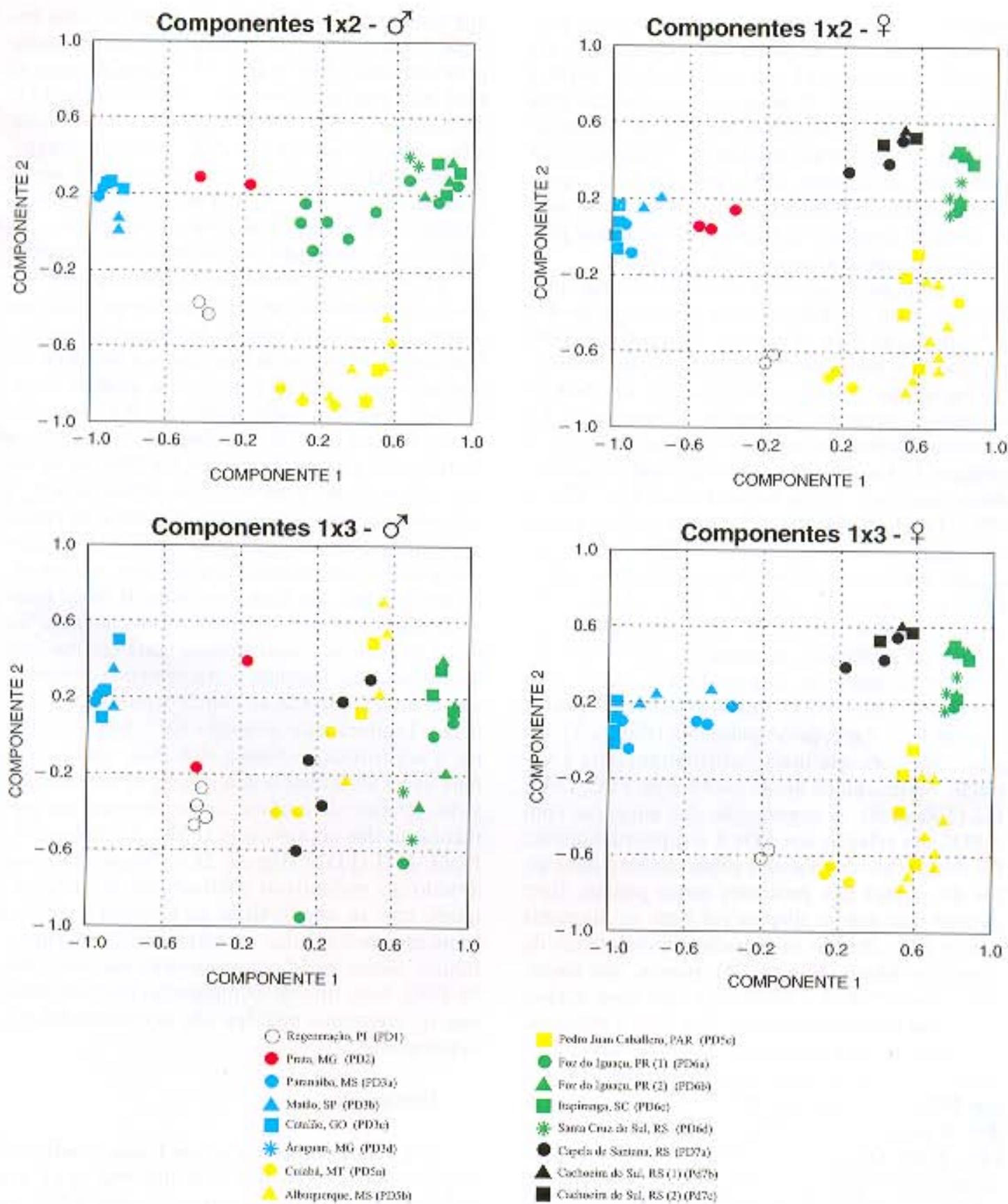

Figura 7 - Gráficos bivariados com as proję̧ues resultantes da analise de componentes principais para machos e fèmeas dus amostras de filhotes do complexo Bothrops neawiedi analisadas. As projeçóes represcntam os três primeiros componentes principais combinados de duas maneiras $(1 \times 2$ e $1 \times 3)$. Branco $=$ PD1; vermelho $=$ PD $2 ; a z u l=$ PD3; amarelo $=$ PDS; verde $=$ PD6; preto $=$ PD7. 
também apresentou um estado exclusivo: pigmentação mais conspícua nas supralabiais da metade anterior da boca (estado 1 do caráter EPSL; Figura 2E). Entretanto, as amostras com os PDs 5 e 6 aparecem separadas ao longo do segundo componente principal, o que deve ser resultante do caráter FPC, que, no PD5, apresentou o estado 0 (faixas pós-cefálicas ausentes) e, no PD6, o estado 2 (faixas pós-cefálicas presentes e bastante alongadas) (Figura $2 \mathrm{~F}$ ).

O terceiro componente principal aparentemente pouco contribuiu para a segregação das amostras com PDs diferentes. De fato, esse eixo representou menos de $14 \%$ da variação observada em ambos os sexos (Tabelas 3 e 4). Para os machos, a principal tendência de separação foi observada entre as amostras com os PDs 1 e 3 (Figura 7). Os três caracteres que mais contribuíram para este componente foram FPC, DA e PSL (Tabela 5). Os dois primeiros não permitem distinguir as amostras com os PDs 1 e 3, enquanto o último é informativo: indivíduos com o PD1 mostraram o estado 2 desse caráter, enquanto aqueles com o PD3 apresentaram o estado 0. Para as fêmeas, as amostras com o PD2 separaram-se daquelas com os PDs 1,3 e 6 , e as amostras com o PD7 destacaram-se daquelas com os PDs 1 e 6, principalmente (Figura 7). Os três caracteres que mais contribuíram para a variação representada nesse eixo foram $\mathrm{FPC}, \mathrm{DA} \mathrm{e}$ D2 (Tabela 6). A segregação das amostras com o PD2 em relação aos PDs 1 e 6 provavelmente foi decorrente dos valores relativamente mais altos do caráter DA presentes nesse padrão. Esse caráter não esteve disponível para as amostras com o PD1 devido às manchas dorsolaterais de contorno difuso (Figura 2B); porém, nas amostras com o PD6 foi observado que esse caráter apresenta baixas contagens. Em outras palavras, verificou-se uma tendência das manchas dorsolaterais encontrarem-se mais próximas entre si nos PDs 1 e 6 que no PD2. Entre as amostras das fêmeas com os PDs 2 e 3 , os caracteres FPC, DA e D2 aparentemente não foram responsáveis pela segregação. Os caracteres BMD, MI 'e MEL, que mostraram certa contribuição para o eixo 3 (Tabela 6) e estados diferentes nos dois padrões, foram os que provavelmente separaram as amostras com os PDs 2 e 3 dessa análise. Nas amostras das fêmeas com o PD7, os caracteres que devem ter contribuído na distinção com relação às amostras com os PDs 1 e 6 foram muito provavelmente FPC e DA. No primeiro caso, o PD1 não possui faixas pós-cefálicas (estado 0 do caráter FPC), o PD6 as possui bastante alongadas (estado 2) e o PD7 apresenta faixas, porém curtas (estado 1). No caso do DA, como já citado anteriormente, os PDs 1 e 6 mostraram tendência de baixas contagens desse caráter em oposição ao observado nas amostras com o PD7.

$\mathrm{O}$ estudo das amostras de filhotes permitiu ainda a constatação da variação ontogenética em alguns aspectos da coloração dessas serpentes. Um desses aspectos, já bastante conhecido e registrado para muitas espécies do gênero, compreende a extremidade mais clara da cauda dos recém-nascidos e jovens, que vai escurecendo ao longo do desenvolvimento. Os filhotes de todas as amostras, sem exceção, apresentaram a extremidade da cauda branca. O outro aspecto é a pigmentação melânica mais intensa dos filhotes quando comparados aos adultos, principalmente no caso das ninhadas onde as mães eram conhecidas (Tabela 1). Dessa forma, as manchas dos filhotes são relativamente mais escuras que as dos adultos. Durante o crescimento, portanto, existe uma tendência ao clareamento da pigmentação. Embora essa variação não chegue a alterar a conformação básica dos PDs, pode algumas vezes dificultar a determinação exata de alguns estados de caráter, como ocorreu na pigmentação das supralabiais (PSL) da ninhada de Prata, MG (PD2) (Figura 2C). Nesses filhotes, devido ao melanismo particularmente intenso, quase não se verificaram as grandes manchas brancas arredondadas características das supralabiais desse padrão e presentes na mãe (IB56.806). Este tipo de comparação revelou ainda que os diferentes padrões são determinados geneticamente.

\section{Discussão}

Diferenças entre os sexos foram confirmadas por caracteres que comumente apontam dimorfismo sexual em serpentes. Em geral, as fêmeas mostraram menor contagem de sub-caudais, maior contagem de ventrais e dorsais e caudas de comprimento menor que os machos. Algumas fêmeas apresentaram também compri- 
mento da cabeça relativamente maior que os machos. Essas tendências, com exceção das cabeças maiores nas fêmeas, já haviam sido registradas em Bothrops neuwiedi pauloensis (Valdujo et al., no prelo) e em B. n. pubescens (Almeida, 1999). Tais tendências são comuns nas serpentes de um modo geral e em várias outras espécies de Bothrops (Leloup, 1975, 1984; Marques, 1998; Sazima, 1992; Sazima e Manzani, 1998; Shine, 1994; Solórzano e Cerdas, 1989). As pressões seletivas que levaram a esse tipo de dimorfismo sexual podem estar relacionadas a benefícios reprodutivos, como o aumento da fecundidade: fêmeas maiores podem ter ninhadas maiores (Seigel e Ford, 1987). O maior comprimento da cauda dos machos, por sua vez, é determinado pela presença dos hemipênis e de seus músculos retratores (King, 1989). Diferenças entre os sexos no tamanho da cabeça geralmente estão relacionadas a diferenças na dieta e no uso de micro-hábitats (Shine, 1986).

Além do dimorfismo sexual, foram detectadas amplas variações individual e geográfica (Figuras 2, 4, 5, 6, 7), corroborando trabalho anterior (Silva, 2000). Além da variabilidade nos caracteres, outro aspecto pode ter influenciado alguns dos resultados. A diferença na representatividade de alguns dos PDs pode ter ocasionado algum desvio, principalmente na análise de componentes principais. Enquanto alguns padrões foram bem amostrados, com 40 ou 47 filhotes (PDs 5 e 6 , respectivamente), os PDs 1 e 2 tiveram, cada um, apenas cinco filhotes amostrados (Tabela 1). Os padrões com as maiores amostras (PDs 5 e 6 ) apresentaram maior variabilidade, como pode ser observado na ampla dispersão dos indivíduos desses PDs nos gráficos da análise de componentes principais (Figura 7). A maior heterogeneidade observada nas amostras com o PD5 (Figura 7, em amarelo) pode ser decorrente da variação geográfica mais intensa. Entre as amostras, aquelas com o PD5 foram as que apresentaram as maiores distâncias geográficas entre si (Figura 1).

Apesar da ampla variabilidade, os caracteres qualitativos revelaram-se relativamente constantes dentro de cada PD e foram os principais responsáveis pela segregação observada entre amostras de PDs diferentes. Tais caracteres podem, portanto, ser considerados bons marca- dores para o diagnóstico desses padrões.

Foi registrada também variação no padrão de colorido ao longo do desenvolvimento: os filhotes apresentaram a extremidade da cauda branca e mostraram-se mais melânicos que os adultos. Mudanças ontogenéticas comuns em serpentes já haviam sido registradas para o complexo $B$. neuwiedi, como variações na dieta (Almeida, 1999; Valdujo et al., no prelo), no veneno (Castro, 1999; Furtado et al., 1991) e na coloração da extremidade da cauda (Almeida, 1999; Leitão-de-Araújo et al., 1998; Melgarejo, 1977), esta última aparentemente relacionada ao comportamento de engodo caudal e à variação ontogenética da dieta (Sazima, 1991, 1992).

Variação ontogenética também foi observada em um dos caracteres morfométricos: as fêmeas das ninhadas não apresentaram comprimento rostro-cloacal maior que os respectivos machos, como observado nos adultos (Silva, 2000). Resultados semelhantes foram obtidos para B. n. pubescens (Almeida, 1999). Isso indica que ambos os sexos nascem com aproximadamente o mesmo comprimento relativo do corpo, mas as fêmeas crescem mais que os machos ao longo do desenvolvimento.

O presente trabalho corroborou os resultados obtidos na revisão recente do complexo $B$. neuwiedi (Silva, 2000), uma vez que foram diagnosticados, entre os filhotes, seis dos sete padrões de desenho observados nos adultos desse grupo. Há evidências, portanto, de que tais padrões são determinados geneticamente e podem corresponder a espécies diferentes desse complexo.

\section{Agradecimentos}

Ao Prof. Dr. Miguel Trefaut Rodrigues, pelas críticas e orientação; a Profa. Dra. Maria de Fátima D. Furtado, chefe da Seção de Herpetologia do Instituto Butantan, por permitir o acesso à coleção de serpentes deste Instituto; ao Prof. Dr. Jaime Bertoluci, pela leitura crítica do manuscrito; a Renata Cecília Amaro, pela ajuda com as figuras; a Tereza Cristina Orlando, pela revisão do texto; à FAPESP, processo $n^{2}$ 98/ 10370-8, pelo apoio financeiro que permitiu a realização deste estudo. 


\section{Referências Bibliográficas}

Almeida, M. T. 1999. História Natural de Bothrops pubescens (Serpentes, Viperidae). Dissertação de Mestrado. Universidade Estadual Paulista "Júlio de Mesquita Filho", Rio Claro, Brasil, 82 pp.

Alves, M. L. M., M. Leitão-de-Araújo e E. Caberlon. 1998. Atividade reprodutiva de Bothrops neuwiedi em cativeiro (Serpentes, Viperidae). Iheringia, Série Zoologia 84: 185-191.

Antonio, F. B. 1980. Mating behavior and reproduction of the eyelash viper (Bothrops schlegeli). Herpetologica 36: 231-233.

Blody, D. A. 1983. Notes on the reproductive biology of the eyelash viper Bothrops schlegeli in captivity. Herpetological Review 14: 45-46.

Calleffo, M. C. 1996. Diferenças sexuais nas contagens de escamas de ninhadas de Crotalus durissus do Brasil. Libro de Resúmenes do IV Congreso Latinoamericano de Herpetología, Santiago, Chile: 107.

Castro, F. T. 1999. Variação Ontogenética de Algumas Atividades do Veneno da Serpente Bothrops neuwiedi diporus do Rio Grande do Sul. Dissertação de Mestrado. Universidade de São Paulo, Brasil. 85 pp.

Crisci, J. V. e M. F. L. Armengol. 1983. Introduccion a la Teoria y Practica de la Taxonomia Numerica. Serie de Biologia, Monografia 28, Secretaria Geral da OEA, Washington. $132 \mathrm{pp}$.

Cruz, G. A., A. Soto e R. V. Bermúdez. 1989. Reproducción de Bothrops asper y $B$. nummifer en Honduras (Serpentes, Viperidae). Revista de Biologia Tropical 37: 201-202.

Federsoni, Jr., P. A. 1980 [1978/79]. Criação e manutenção de serpentes da espécie Bothrops atrox nascidas em cativeiro (Serpentes - Viperidae - Crotalinae). Memórias do Instituto Butantan 42/43; 159-169.

Furtado, M. F. D., M. Maruyama, A. S. Kamiguti e L. C. Antonio. 1991. Comparative study of nine Bothrops snake venoms from adult female snakes and their offspring. Toxicon 29: 219-226.

Hoge, A. R. e P. A. Federsoni Jr. 1978 [1976/77]. Observações sobre uma ninhada de Bothrops atrox (Linnaeus, 1758) [Serpentes: Viperidae: Crotalinae]. Memórias do Instituto Butantan 40/41: 19-36.

James, F. C. e C. E. Mcculloch. 1990. Multivariate analysis in ecology and systematics: Panacea or Pandora's Box? Annual Review Ecology and Systematics 21: 129-166.

King, R. B. 1989. Sexual dimorphism in snake tail lenght: sexual selection, natural selection, or morphological constraint? Biological Journal of the Linnean Society 38: $133-154$.

Leitão-de-Araújo, M., M. L. M. Alves, R. L. Balestrin e L. F. S. Aguiar. 1998. Nascimento e desenvolvimento de Bothrops neuwiedi em cativeiro (Serpentes, Viperidae). Theringia, Série Zoologia 85: 3-10.
Leitão-de-Araújo, M. e L. A. Matschulat-ely. 1980. Notas sobre a biologia de tanatofídios criados em cativeiro $2^{\star}$ parte. (Ophidia - Elapidae e Viperidae). Iheringia, Série Zoologia 55: 9-26.

Leitāo-de-Araújo, M. e M. Perazzolo. 1974. Nota prévia sobre a biologia de tanatofídios em cativeiro (Ophidia, Elapidae e Viperidae). Iheringia, Série Zoologia 45: 55-66.

Leloup, P. 1973. Essais de rationalisation dans le maintien d'un serpentarium à but industriel. Acta Tropica 30: 281-311.

Leloup, P. 1975. Observations sur la reproduction de Bothrops moojeni Hoge en captivité. Acta Zoologica et Pathologica Antverpiensia 62: 173-201.

Leloup, P. 1984. Various aspects of venomous snake breeding in large scale. Acta Zoologica et Pathologica Antverpiensia 78: 177-198.

Marques, O. A. V. 1998. Reprodução e hábitos alimentares de Bothrops jararacussu (Serpentes, Viperidae), na Estaçāo Ecológica Juréia-Itatins, litoral sul de São Paulo. Resumos do XXII Congresso Brasileiro de Zoologia, Recife: 271 .

Melgarejo, A, R, 1977. Observaciones sobre nacimiento en el laboratorio de Bothrops neuwiedi pubescens (Cope, 1870) (Ophidia, Crotalinae). Revista de Biologia del Uruguay 5: 35-41.

Murphy, J. B. e L. A. Mitchell. 1984. Miscellaneous notes on the reproductive biology of reptiles. 6 . Thirteen varieties of the genus Bothrops (Serpentes, Crotalidae). Acta Zoologica et Pathologica Antverpiensia 78: 199. 214.

Peñaranda, E. B. 1995. Bothrops neuwiedi (Yoperojobobo, Neuwied's Lancehead). Brood size. Herpetological Review 26: 205-206.

Pezzano, V. 1986. Reproduction of Bothrops alternatus (Dumèril, Bibron e Dumèril, 1854) in captivity. Litteratura Serpentium 6: 13-18.

Sazima, 1. 1991. Caudal luring in two neotropical pitvipers, Bothrops jararaca and Bothrops jararacussu. Copeia I: $245-248$.

Sazima, 1. 1992. Natural history of the jararaca pitviper, Bothrops jararaca, in southeastern Brazil. In: Campbell, J. A. e E. D. BRODIE Jr. (eds.). Biology of the Pitvipers. Selva, Tyler, Texas. Pp. 99-216.

Sazima, I, e P. R. Manzani, 1998. Bothrops fonsecai (Fonseca's Lancehead). Reproduction and size. Herpetological Review 29: 102-103.

Seigel, R. A. e N. B. Ford. 1987. Reproductive ecology. In Seigel, R. A., J. T. Collins e S. S. Novak (eds.). Snakes: ecology and evolutionary biology. McGrawHill and Company, New York. Pp. 210-252.

Shine, R. 1986. Sexual differences in morphology and niche utilization in aquatic snake, Acrochordus arafurae. Oecologia 69: 260-267. 
Shine, R. 1994. Sexual size dimorphism in snakes revisited. Copeia 2: 326-346.

Silva, V. X. 2000. Revisão Sistemática do Complexo Bothrops neuwiedi (Serpentes, Viperidae, Crotalinae). 2 vols. Tese de Doutorado. Universidade de São Paulo, Brasil.

Solórzano, A. 1989. Distribución y aspectos reproductivos de la mano de piedra, Bothrops nummifer (Serpentes: Viperidae), en Costa Rica. Revista de Biologia Tropical 37: 133-137.

Solórzano, A. e L. Cerdas. 1989. Reproductive biology and distribution of the terciopelo, Bothrops asper Garman (Serpentes: Viperidae), in Costa Rica. Herpetologica 45: 195-207.

Systat, Inc, 1993. SYSTAT for Windows. Version 5,03. 1990-1992. Evanston, Illinois.

Thorpe, R. S. 1976. Biometric analysis of geographic variation and racial affinities. Biological Reviews 51: $407-452$
Thorpe, R. S. 1980. A comparative study of ordination techniques in numerical taxonomy in relation to racial variation in the ringed snake Natrix natrix (L.). Biological Journal of the Linnean Society 13: 7-40.

Valdujo, P. H., C. E. Nogueira e M. Martins. No prelo. Ecology of Bothrops neuwiedi pauloensis (Serpentes: Viperidae: Crotalinae) in the Brazilian Cerrado. Journal of Herpetology.

Vanzolini, P. E. 1993. Métodos Estatísticos Elementares em Sistemática Zoológica. Editora Hucitec, São Paulo. $130 \mathrm{pp}$.

Wüster, W., R. S. Thorpe, G. Puorto, M. F. D. Furtado, S. A. Hoge, M. G. Salomão, R. D. G. Theakston e D. A. Warrell. 1996. Systematics of the Bothrops atrox complex (Reptilia: Serpentes: Viperidae) in Brazil: a multivariate analysis. Herpetologica 52; 263-271.

Zar, J. H. 1984. Biostatistical Aanalysis. 2nd ed. PrenticeHall. Englewood Cliffs, New Jersey. 718 pp.

\section{Apêndice I}

Material examinado da coleção do Instituto Butantan (IB); F1 a F10 = filhotes sem numeração da fêmea prenhe IB-7.004:

Machos - BRASIL: Goiás: Catalão: IB-32.788 a 32.790. Mato Grosso: Cuiabá: IB-57.066, IB-57.067, IB-57.071, IB-57.073, IB-57.075, IB-57.077. Mato Grosso do Sul: Albuquerque: IB-4.880, IB-4.881, IB-4.884, IB-4.886, IB-4.887, IB4.893, IB-4.895, IB-4.898, IB-4.900; Paranaíba: IB-46.126, IB-46.131, IB-46.136, IB-46.139. Minas Gerais: Araguari: IB-56.528, IB-56.531; Prata: IB-56.809, IB-56.811. Paraná: Foz do Iguaçu: IB-25.808, IB-25.810, IB-25.813 a 25.815 , IB-27.061 a 27.066 , IB-27.069, IB27.070, IB-27.072 a 27.074, IB-27.078. Piauí: Regeneração: IB-562, IB-565, IB-3.100. Rio Grande do Sul: Cachoeira do Sul: IB-28.907; Capela de Santana: F1, F4, F8 a 10; Santa Cruz do Sul: IB-15.307, IB-15.308, IB-15.311, IB15.313, IB-15.316, IB-15.317, IB-15.319. Santa Catarina: Itapiranga: IB-27.140, IB-27.142, IB27.144. São Paulo: Matão: IB-21.160, IB21.161, IB-21.165. PARAGUAI: Amambay: Pedro Juan Caballero: IB-40.637 a 40.639 , IB40.643 .
Fêmeas - BRASIL: Goiás: Catalão: IB32.768, IB-32.775, IB-32.781, IB-32.785. Mato Grosso: Cuiabá: IB-57.074, IB-57.076, IB57.078. Mato Grosso do Sul: Albuquerque: IB4.877 a 4.879 , IB-4.882, IB-4.883, IB-4.885, IB-4.888, IB-4.890, IB-4.891, IB-4.892, IB4.894, IB-4.896, IB-4.897, IB-4.899; Paranaíba: IB-46.127, IB-46.129, IB-46.135, IB-46.138. Minas Gerais: Araguari: IB-56.529, IB-56.530, IB-56.532, IB-56.533; Prata: IB-56.807, IB56.808, IB-56.810. Paraná: Foz do Iguaçu: IB25.811 , IB-25.812, IB-27.067, IB-27.068, IB27.071, IB-27.075 a 27.077. Piauí: Regeneração: IB-566, IB-567. Rio Grande do Sul: Cachoeira do Sul: IB-28.972, IB-28.974, IB32.361, IB-32.758; Capela de Santana: F2, F3, F5 a 7; Santa Cruz do Sul: IB-15.306, IB15.309 , IB-15.310, IB-15.312, IB-15.314, IB15.315, IB-15.318, IB-15.320. Santa Catarina: Itapiranga: IB-27.138, IB-27.139, IB-27.141, IB-27.143. São Paulo: Matão: IB-21.159, IB21.162 a 21.164 , IB-21.166. PARAGUAI: Amambay: Pedro Juan Caballero: IB-40.636, IB-40.640 a 40.642 . 


\section{Apêndice II}

Estatística descritiva do comprimento da cabeça $(\mathrm{CAB})$ para machos e fêmeas das 16 amostras de filhotes do complexo Bothrops neuwiedi. $\mathrm{PD}=$ padrão de desenho; $\mathrm{A}=$ amplitude; $\mathrm{X} \pm \mathrm{s}_{\mathrm{x}}=$ média \pm desvio padrão da média; $s=$ desvio padrão; $C V=$ coeficiente de variação; $I=$ intervalo de confiança da média. As letras que acompanham alguns PDs discriminam as diferentes amostras que apresentaram o mesmo PD.

\begin{tabular}{|c|c|c|c|c|c|c|c|c|}
\hline Amostras & PD & & $\mathbf{N}$ & $\mathbf{A}(\mathrm{mm})$ & $\mathbf{X} \pm \mathbf{s}_{\mathbf{x}}(\mathrm{mm})$ & $\mathbf{s}(\mathbf{m m})$ & CV (\%) & I (mm) \\
\hline \multirow[t]{2}{*}{ Regeneração, PI } & 1 & $\sigma^{x}$ & 3 & $13,40-17,50$ & $15,53 \pm 1,26$ & 2,18 & 14,0 & $13,02-18,05$ \\
\hline & & & 2 & $15,70-16,10$ & $15,90 \pm 0,20$ & 0,28 & 1,8 & $15,50-16,36$ \\
\hline \multirow{2}{*}{ Prata, MG } & 2 & $\sigma^{x}$ & 2 & $14,10-15,25$ & $14,67 \pm 0,57$ & 0,81 & 5,5 & $13,53-15,83$ \\
\hline & & 우 & 3 & $14,45-15,75$ & $15,07 \pm 0,38$ & 0,65 & 4,3 & $14,31-15,82$ \\
\hline \multirow[t]{2}{*}{ Paranaíba, MS } & $3 \mathrm{a}$ & $\sigma^{\pi}$ & 3 & $13,55-14,35$ & $13,97 \pm 0,23$ & 0,40 & 2,9 & $13,50-14,43$ \\
\hline & & q & 3 & $14,00-14,55$ & $14,33 \pm 0,17$ & 0,29 & 2,0 & $13,99-14,67$ \\
\hline \multirow{2}{*}{ Matão, SP } & $3 b$ & $\sigma^{\pi}$ & 3 & $14,60-15,55$ & $15,17 \pm 0,29$ & 0,50 & 3,3 & $14,59-15,75$ \\
\hline & & 우 & 5 & $12,80-16,35$ & $14,84 \pm 0,63$ & 1,40 & 9,4 & $13,59-16,09$ \\
\hline \multirow[t]{2}{*}{ Catalão, GO } & $3 \mathrm{c}$ & $\sigma^{x}$ & 3 & $13,15-13,40$ & $13,27 \pm 0,07$ & 0,13 & 0,9 & $13,12-13,41$ \\
\hline & & 우 & 4 & $12,95-13,50$ & $13,25 \pm 0,12$ & 0,23 & 1,8 & $13,02-13,48$ \\
\hline \multirow[t]{2}{*}{ Araguari, MG } & $3 \mathrm{~d}$ & $\sigma^{x}$ & 2 & $14,05-15,35$ & $14,70 \pm 0,65$ & 0,92 & 6,3 & $13,40-16,00$ \\
\hline & & 우 & 4 & $13,95-15,20$ & $14,56 \pm 0,25$ & 0,51 & 3,5 & $14,05-15,07$ \\
\hline \multirow{2}{*}{ Cuiabá, MT } & $5 a$ & $\sigma^{x}$ & 6 & $14,30-15,40$ & $14,86 \pm 0,16$ & 0,39 & 2,6 & $14,54-15,17$ \\
\hline & & of & 3 & $14,10-14,55$ & $14,30 \pm 0,13$ & 0,23 & 1,6 & $14,04-14,56$ \\
\hline \multirow[t]{2}{*}{ Albuquerque,MS } & $5 b$ & $\sigma^{x}$ & 9 & $12,30-16,00$ & $14,46 \pm 0,35$ & 1,07 & 7,4 & $13,75-15,17$ \\
\hline & & q & 13 & $13,75-16,25$ & $15,03 \pm 0,25$ & 0,89 & 5,9 & $14,54-15,52$ \\
\hline \multirow[t]{2}{*}{ Pedro Juan Caballero, PAR } & $5 c$ & $\sigma^{x}$ & 4 & $15,65-16,25$ & $16,00 \pm 0,13$ & 0,25 & 1,6 & $15,75-16,25$ \\
\hline & & 우 & 4 & $16,10-16,75$ & $16,43 \pm 0,17$ & 0,35 & 2,1 & $16,08-16,77$ \\
\hline \multirow{2}{*}{ Foz do Iguaçu, PR (1) } & $6 a$ & $\sigma^{*}$ & 5 & $15,20-17,15$ & $16,33 \pm 0,31$ & 0,71 & 4,3 & $15,70-16,96$ \\
\hline & & 우 & 2 & $16,55-17,20$ & $16,87 \pm 0,33$ & 0,46 & 2,7 & $16,23-17,53$ \\
\hline \multirow{2}{*}{ Foz do Iguaçu, PR (2) } & $6 \mathrm{~b}$ & $\sigma^{x}$ & 12 & $11,45-17,75$ & $16,02 \pm 0,49$ & 1,69 & 10,6 & $15,04-16,99$ \\
\hline & & 우 & 6 & $15,90-17,25$ & $16,67 \pm 0,21$ & 0,51 & 3,1 & $16,26-17,09$ \\
\hline \multirow[t]{2}{*}{ Itapiranga, SC } & $6 c$ & $\sigma^{x}$ & 3 & $16,60-17,45$ & $17,00 \pm 0,25$ & 0,43 & 2,5 & $16,51-17,49$ \\
\hline & & 우 & 4 & $17,30-18,65$ & $17,90 \pm 0,29$ & 0,58 & 3,2 & $17,32-18,48$ \\
\hline \multirow[t]{2}{*}{ Santa Cruz do Sul, RS } & $6 \mathrm{~d}$ & $\sigma^{x}$ & 7 & $16,00-18,00$ & $16,83 \pm 0,27$ & 0,71 & 4,2 & $16,29-17,36$ \\
\hline & & 우 & 8 & $16,10-18,05$ & $17,01 \pm 0,25$ & 0,70 & 4,1 & $16,52-17,51$ \\
\hline \multirow[t]{2}{*}{ Capela de Santana, RS } & $7 \mathrm{a}$ & $\sigma^{x}$ & 5 & $12,85-14,15$ & $13,46 \pm 0,25$ & 0,56 & 4,2 & $12,96-13,96$ \\
\hline & & 우 & 5 & $12,55-14,30$ & $13,75 \pm 0,32$ & 0,71 & 5,2 & $13,11-14,39$ \\
\hline \multirow[t]{2}{*}{ Cachoeira do Sul, RS (1) } & $7 b$ & $0^{x}$ & 1 & 15,05 & 15,050 & - & - & - \\
\hline & & 우 & 2 & $17,20-17,25$ & $17,22 \pm 0,03$ & 0,03 & 0,2 & $17,17-17,27$ \\
\hline \multirow[t]{2}{*}{ Cachoeira do Sul, RS (2) } & $7 \mathrm{c}$ & $0^{x}$ & 0 & - & - & - & - & - \\
\hline & & 우 & 2 & $16,05-16,35$ & $16,20 \pm 0,15$ & 0,21 & 1,3 & $15,90-16,50$ \\
\hline
\end{tabular}




\section{Apêndice III}

Estatística descritiva do comprimento rostro-cloacal (CRC) para machos e fêmeas das 16 amostras de filhotes do complexo Bothrops neuwiedi. $\mathrm{PD}=$ padrão de desenho; $\mathrm{A}=$ amplitude; $\mathrm{X} \pm \mathrm{s}_{\mathrm{x}}=$ média \pm desvio padrão da média; $s=$ desvio padrão; $C V=$ coeficiente de variação $(\%) ; \mathrm{I}=$ intervalo de confiança da média. As letras que acompanham alguns PDs discriminam as diferentes amostras que apresentaram o mesmo PD.

\begin{tabular}{|c|c|c|c|c|c|c|c|c|}
\hline Amostras & PD & $\mathrm{S}$ & $\mathbf{N}$ & $\mathbf{A}$ & $X+s_{x}$ & s & CV $(\%)$ & I \\
\hline \multirow[t]{2}{*}{ Regeneração, PI } & 1 & $\sigma^{x}$ & 3 & $205-256$ & $238,7 \pm 16,8$ & 29,2 & 12,2 & $205,0-272,4$ \\
\hline & & 우 & 2 & $255-275$ & $265,0+10,0$ & 14,1 & 5,3 & $245,0-285,0$ \\
\hline \multirow[t]{2}{*}{ Prata, MG } & 2 & $\sigma^{x}$ & 2 & $225-226$ & $225,5 \pm 0,5$ & 0,7 & 0,3 & $224,5-226,5$ \\
\hline & & 우 & 3 & $212-224$ & $219,7 \pm 3,8$ & 6,7 & 3,0 & $212,0-227,3$ \\
\hline \multirow{2}{*}{ Paranaíba, MS } & $3 \mathrm{a}$ & $\sigma^{x}$ & 4 & $210-242$ & $229,5 \pm 7,3$ & 14,5 & 6,3 & $215,0-244,0$ \\
\hline & & 우 & 4 & $215-231$ & $223,2 \pm 4,0$ & 7,9 & 3,6 & $215,6-231,4$ \\
\hline \multirow[t]{2}{*}{ Matão, SP } & $3 b$ & $\sigma^{x}$ & 3 & $227-243$ & $234,3 \pm 4,7$ & 8,1 & 3,4 & $225,0-243,7$ \\
\hline & & 우 & 5 & $185-243$ & $214,8+10,8$ & 24,2 & 11,3 & $193,1-236,5$ \\
\hline \multirow[t]{2}{*}{ Catalão, GO } & $3 c$ & $\sigma^{x}$ & 3 & $199-204$ & $201,3 \pm 1,4$ & 2,5 & 1,2 & $198,4-204,2$ \\
\hline & & 우 & 4 & $193-200$ & $196,5+1,5$ & 3,1 & 1,6 & $193,4-199,6$ \\
\hline \multirow[t]{2}{*}{ Araguari, MG } & $3 d$ & $\sigma^{\prime}$ & 2 & $201-210$ & $205,5 \pm 4,5$ & 6,4 & 3,1 & $196,5-214,5$ \\
\hline & & 우 & 4 & $197-214$ & $205,7+3,7$ & 7,4 & 3,6 & $198,4-213,1$ \\
\hline \multirow[t]{2}{*}{ Cuiabá, MT } & $5 \mathrm{a}$ & $\sigma^{x}$ & 6 & $215-232$ & $223,7 \pm 2,7$ & 6,6 & 2,9 & $218,3-229,1$ \\
\hline & & 우 & 3 & $216-220$ & $218,0+1,1$ & 2,0 & 0,9 & $215,7-220,3$ \\
\hline \multirow[t]{2}{*}{ Albuquerque,MS } & $5 b$ & $\sigma^{x}$ & 9 & $173-227$ & $203,9 \pm 5,7$ & 17,2 & 8,4 & $192,4-215,3$ \\
\hline & & 와 & 12 & $175-216$ & $202,6+3,9$ & 13,5 & 6,7 & $194,8-210,4$ \\
\hline \multirow{2}{*}{ Pedro Juan Caballero, PAR } & $5 c$ & or & 4 & $216-231$ & $224,7 \pm 3,5$ & 7,1 & 3,2 & $217,7-231,8$ \\
\hline & & 우 & 4 & $224-233$ & $230,5+2,2$ & 4,4 & 1,9 & $226,1-234,9$ \\
\hline \multirow[t]{2}{*}{ Foz do Iguaçu, PR (1) } & $6 a$ & $\sigma^{x}$ & 5 & $200-259$ & $237,8 \pm 10,0$ & 22,3 & 9,4 & $217,9-257,7$ \\
\hline & & 우 & 2 & $243-250$ & $246,5+3,5$ & 4,9 & 2,0 & $239,5-253,5$ \\
\hline \multirow[t]{2}{*}{ Foz do Iguaçu, PR (2) } & $6 b$ & $\sigma^{x}$ & 12 & $231-283$ & $259,5 \pm 5,1$ & 17,7 & 6,8 & $249,3-269,7$ \\
\hline & & 우 & 6 & $236-272$ & $259,8+6,2$ & 10,1 & 5,8 & $247,5-272,2$ \\
\hline \multirow[t]{2}{*}{ Itapiranga, SC } & $6 c$ & $\sigma^{x}$ & 3 & $263-281$ & $270,3 \pm 5,5$ & 9,5 & 3,5 & $259,4-281,3$ \\
\hline & & 우 & 4 & $271-285$ & $275,7+3,2$ & 6,4 & 2,3 & $269,3-282,1$ \\
\hline \multirow[t]{2}{*}{ Santa Cruz do Sul, RS } & $6 \mathrm{~d}$ & $\sigma^{\prime \prime}$ & 7 & $270-290$ & $280,0 \pm 2,8$ & 7,5 & 2,7 & $274,3-285,7$ \\
\hline & & 우 & 8 & $250-282$ & $270,0+3,4$ & 9,7 & 3,6 & $263,1-276,9$ \\
\hline \multirow[t]{2}{*}{ Capela de Santana, RS } & $7 \mathrm{a}$ & $\sigma^{\pi}$ & 5 & $189-202$ & $193,8 \pm 2,3$ & 5,1 & 2,6 & $191,5-198,3$ \\
\hline & & 우 & 5 & $181-192$ & $186,6+2,0$ & 4,4 & 2,4 & $182,7 \quad 190,5$ \\
\hline \multirow[t]{2}{*}{ Cachoeira do Sul, RS (1) } & $7 b$ & $\sigma^{7}$ & 1 & 246 & 246,0 & - & - & - \\
\hline & & 우 & 2 & $245-271$ & $258,0 \pm 13,0$ & 18,4 & 7,1 & $232,0-284,0$ \\
\hline \multirow[t]{2}{*}{ Cachoeira do Sul, RS (2) } & $7 \mathrm{c}$ & $\sigma^{x}$ & 0 & - & - & - & - & - \\
\hline & & q & 2 & $242-243$ & $242,5 \pm 0,5$ & 0,7 & 0,3 & $241,5-243,5$ \\
\hline
\end{tabular}




\section{Apêndice IV}

Estatística descritiva do comprimento da cauda (CAU) para machos e fêmeas das 16 amostras de filhotes do complexo Bothrops neuwiedi. $\mathrm{PD}=$ padrão de desenho; $\mathrm{A}=$ amplitude; $\mathrm{X} \pm \mathrm{s}_{\mathrm{x}}=$ média \pm desvio padrão da média; $\mathrm{s}=$ desvio padrão; $\mathrm{CV}=$ coeficiente de variação $(\%)$; $=$ intervalo de confiança da média. As letras que acompanham alguns PDs discriminam as diferentes amostras que apresentaram o mesmo PD.

\begin{tabular}{|c|c|c|c|c|c|c|c|c|}
\hline Amostras & PD & & $\mathbf{N}$ & $\mathbf{A}$ & $\mathbf{X}+\underline{s}_{x}$ & $\mathbf{s}$ & CV (\%) & I \\
\hline \multirow[t]{2}{*}{ Regeneração, PI } & 1 & $\sigma^{x}$ & 3 & $32-36$ & $34,3 \pm 1,2$ & 2,1 & 6,1 & $31,9-36,7$ \\
\hline & & ㅇ & 2 & $31-33$ & $32,0 \pm 1,0$ & 1,4 & 4,4 & $30,0-34,0$ \\
\hline Prata, MG & 2 & $\sigma^{x}$ & 2 & $35-37$ & $36,0 \pm 1,0$ & 1,4 & 3,9 & $34,0-38,0$ \\
\hline \multirow[t]{2}{*}{ Paranaíba, MS } & & 우 & 3 & $30-31$ & $30,3 \pm 0,3$ & 0,6 & 1,9 & $29,7-31,0$ \\
\hline & $3 a$ & $\begin{array}{l}0 \\
0\end{array}$ & 4 & $\begin{array}{l}36-39 \\
30-32\end{array}$ & & 1,4 & 3,8 & $35,6-38,4$ \\
\hline \multirow[t]{2}{*}{ Matão, SP } & & $a^{+}$ & $\begin{array}{l}4 \\
3\end{array}$ & $\begin{array}{l}30-32 \\
33-41\end{array}$ & $\begin{array}{l}31,2 \pm 0,5 \\
35,7+2,7\end{array}$ & 1,0 & 3,1 & $\begin{array}{l}30,3-32,2 \\
303-410\end{array}$ \\
\hline & $3 b$ & 우 & 5 & $23-36$ & & $\begin{array}{l}4,6 \\
5,4\end{array}$ & $\begin{array}{l}12,9 \\
18,6\end{array}$ & $\begin{array}{l}30,3-41,0 \\
24,0-33,6\end{array}$ \\
\hline \multirow[t]{2}{*}{ Catalão, GO } & $3 \mathrm{c}$ & $\sigma^{x}$ & 3 & $28-32$ & $30,3 \pm 1,2$ & 2,1 & 6,9 & $27,9-32,7$ \\
\hline & & q & 4 & $23-27$ & $25,3 \pm 1,0$ & 2,1 & 8,2 & $23,2-27,3$ \\
\hline \multirow[t]{2}{*}{ Araguari, MG } & $3 d$ & $\sigma^{x}$ & 2 & $30-33$ & $31,5 \pm 1,5$ & 2,1 & 6,7 & $28,5-34,5$ \\
\hline & & q & 4 & $26-30$ & $28,0 \pm 0,9$ & 1,8 & 6,5 & $26,2-29,8$ \\
\hline \multirow[t]{2}{*}{ Cuiabá, MT } & $5 a$ & $\sigma^{*}$ & 6 & $31-35$ & $32,8 \pm 0,7$ & 1,6 & 4,9 & $31,5-34,1$ \\
\hline & & q & 3 & $21-30$ & $27,0 \pm 3,0$ & 5,2 & 19,2 & $21,0-33,0$ \\
\hline \multirow{2}{*}{ Albuquerque, MS } & $5 b$ & $\sigma^{x}$ & 9 & $32-40$ & $36,0 \pm 0,9$ & 2,7 & 7,6 & $34,2-37,8$ \\
\hline & & q & 13 & $28-36$ & $32,6 \pm 0,6$ & 2,2 & 6,8 & $31,4-33,9$ \\
\hline Pedro Juan Caballero, PAR & $5 c$ & $\sigma^{x}$ & 4 & $29-38$ & $34,5 \pm 2,0$ & 4,0 & 11,7 & $30,5-38,5$ \\
\hline \multirow{2}{*}{ Foz do Iguaçu, PR (1) } & $6 a$ & $\begin{array}{l}\text { of } \\
\sigma^{n}\end{array}$ & 4 & $30-33$ & $\begin{array}{l}31,5 \pm 0,9 \\
378+22\end{array}$ & $\begin{array}{l}1,7 \\
5,0\end{array}$ & $\begin{array}{c}5,5 \\
13,3\end{array}$ & $\begin{array}{l}29,8-33,2 \\
33,3-42,3\end{array}$ \\
\hline & & $\begin{array}{l}0^{x} \\
\text { o }\end{array}$ & $\begin{array}{l}5 \\
2\end{array}$ & $\begin{array}{l}29-41 \\
34-36\end{array}$ & $\begin{array}{l}37,8 \pm 2,2 \\
35,0 \pm 1,0\end{array}$ & 1,4 & 4,0 & $\begin{array}{l}33,3-42,3 \\
33,0-37,0\end{array}$ \\
\hline \multirow{2}{*}{ Foz do Iguaçu, PR (2) } & $6 \mathrm{~b}$ & $\sigma^{x}$ & 12 & $35-45$ & $38,7 \pm 0,9$ & 3,2 & 8,3 & $36,8-40,5$ \\
\hline & & 우 & 6 & $30-36$ & $34,0 \pm 0,6$ & 2,1 & 6,2 & $32,3-35,7$ \\
\hline \multirow[t]{2}{*}{ Itapiranga, SC } & $6 \mathrm{c}$ & $\sigma^{x}$ & 3 & $35-40$ & $36,7 \pm 1,7$ & 2,9 & 7,9 & $33,3-40,0$ \\
\hline & & 우 & 4 & $30-36$ & $34,5 \pm 1,5$ & 3,0 & 8,7 & $31,5-37,5$ \\
\hline \multirow{2}{*}{ Santa Cruz do Sul, RS } & $6 \mathrm{~d}$ & $\sigma^{*}$ & 7 & $41-47$ & $45,3 \pm 0,8$ & 2,2 & 4,9 & $43,6-47,0$ \\
\hline & & 우 & 8 & $35-39$ & $37,3 \pm 0,4$ & 1,2 & 3,1 & $36,4-38,1$ \\
\hline Capela de Santana, RS & $7 a$ & $\sigma^{x}$ & 5 & $31-34$ & $32,2 \pm 0,6$ & 1,3 & 4,0 & $31,0-33,4$ \\
\hline \multirow[t]{2}{*}{ Cachoeira do Sul, RS (1) } & & + & 5 & $27-32$ & $\begin{array}{c}29,0 \pm 0,8 \\
40,0\end{array}$ & $\begin{array}{l}1,9 \\
-\end{array}$ & $\begin{array}{c}6,5 \\
-\end{array}$ & $\begin{array}{c}27,3-30,7 \\
-\end{array}$ \\
\hline & $7 \mathrm{~b}$ & $\begin{array}{l}0 \\
\text { q }\end{array}$ & $\begin{array}{l}1 \\
2\end{array}$ & $\begin{array}{c}40 \\
32-41\end{array}$ & $\begin{array}{c}40,0 \\
36,5 \pm 4,5\end{array}$ & 6,4 & 17,4 & $27,5-45,5$ \\
\hline \multirow[t]{2}{*}{ Cachoeira do Sul, RS (2) } & $7 \mathrm{c}$ & $\sigma^{x}$ & 0 & - & - & - & - & - \\
\hline & & q & 2 & $33-33$ & 33,0 & 0 & 0 & $33,0-33,0$ \\
\hline
\end{tabular}

